\title{
A CHARACTERIZATION OF BANACH SPACES CONTAINING $c_{0}$
}

\author{
HASKELL ROSENTHAL
}

\section{Contents}

1. Introduction

2. Permanence properties of (s.s.)-sequences

3. Differences of bounded semi-continuous functions

4. Proof of the main theorem

References

\section{INTRODUCTION}

In 1974, the following subsequence dichotomy was established by the author for real scalars [R1], and refined by L.E. Dor to cover the case of complex scalars [Do] (cf. also [R2] for a general exposition).

Theorem 1.0. Every bounded sequence in a real or complex Banach space has either a weak-Cauchy subsequence, or a subsequence equivalent to the standard $\ell^{1}$-basis.

In this article, I obtain a subsequence principle characterizing spaces containing $c_{0}$, in the same spirit as the above $\ell^{1}-$ Theorem. The principle requires the following new concept:

Definition 1.1. A sequence $\left(b_{j}\right)$ in a Banach space is called strongly summing (s.s.) if $\left(b_{j}\right)$ is a weak-Cauchy basic sequence so that whenever scalars $\left(c_{j}\right)$ satisfy $\sup _{n}\left\|\sum_{j=1}^{n} c_{j} b_{j}\right\|<\infty$, then $\sum c_{j}$ converges.

The following result is the main concern of this article. (A weak-Cauchy sequence is called non-trivial if it is non-weakly convergent.)

Received by the editors October 15, 1992 and, in revised form, July 13, 1993.

1991 Mathematics Subject Classification. Primary 46B03, 46B25; Secondary 04A15.

Key words and phrases. Weakly sequentially complete dual, convex block basis, the $\ell^{1}-$ Theorem, differences of semi-continuous functions.

This research was partially supported by NSF DMS-8903197 and TARP 235. 
Theorem 1.1. Every non-trivial weak-Cauchy sequence in a (real or complex) Banach space has either a strongly summing subsequence or a convex block basis equivalent to the summing basis.

To prove this result, I develop various permanence properties of strongly summing sequences. I also give some new invariants for general discontinuous functions, namely their transfinite oscillations. These are similar to some previous invariants given by A.S. Kechris and A. Louveau [KL]. They are used (in a manner similar to the earlier work in $[\mathrm{KL}]$ ) to characterize differences of bounded semi-continuous functions, which enter into the demonstration of Theorem 1.1 in an essential way. The core of the proof is then a real-variables theorem for obtaining subsequences of a sequence of continuous functions converging pointwise to a function which is not such a difference.

I have attempted to write the rest of this section so as to be accessible to the general mathematical public. Afterwards, I shall freely use standard Banach space facts and terminology. Here is a quick review of some necessary concepts: $c_{0}$ denotes the Banach space of sequences tending to zero, under the sup norm; $\ell^{1}$ the Banach space of absolutely summable sequences, under the norm given by the sum of the absolute-values of the coordinates. A sequence $\left(b_{j}\right)$ of elements of a Banach space $B$ is called a weak-Cauchy sequence if it is a Cauchy sequence in $B$ endowed with the weak topology; equivalently, if $\lim _{j \rightarrow \infty} b^{*}\left(b_{j}\right)$ exists for all $b^{*} \in B^{*}$, the dual of $B .\left(b_{j}\right)$ is called a basic sequence if it is a basis for its closed linear span $\left[b_{j}\right]$; that is, for every $b$ in $\left[b_{j}\right]$, there is a unique sequence of scalars $\left(c_{j}\right)$ so that $b=\sum c_{j} b_{j}$. Given $\left(b_{j}\right)$ a sequence in a Banach space $B$, a sequence $\left(u_{j}\right)$ of non-zero elements of $B$ is called a block basis of $\left(b_{j}\right)$ if there exist integers $0 \leq n_{1}<n_{2}<\cdots$ and scalars $c_{1}, c_{2}, \ldots$ so that $u_{j}=\sum_{i=n_{j}+1}^{n_{j+1}} c_{i} b_{i}$ for all $j=1,2, \ldots ;\left(u_{j}\right)$ is called a convex block basis if the $c_{i}$ 's satisfy: $c_{i} \geq 0$ for all $i$ and $\sum_{i=n_{j}+1}^{n_{j+1}} c_{i}=1$ for all $j$. A standard elementary result yields that if $\left(b_{j}\right)$ is a basic sequence, so is any block basis $\left(u_{j}\right)$. It is evident that if $\left(b_{j}\right)$ is a non-trivial weakCauchy sequence, then so is any convex block basis $\left(u_{j}\right)$ of $\left(b_{j}\right)$. Another standard result (reproved in Section 2) asserts that any non-trivial weak-Cauchy sequence contains a basic subsequence. Given $X$ and $Y$ Banach spaces, a bounded linear operator $T: X \rightarrow Y$ is called an isomorphism between $X$ and $Y$ if $T$ is invertible; equivalently (by the open mapping theorem), if $T$ is oneto-one and onto. If $\left(x_{j}\right)$ and $\left(y_{j}\right)$ are sequences in Banach spaces $X$ and $Y$ respectively, $\left(x_{j}\right)$ and $\left(y_{j}\right)$ are called equivalent if there exists an isomorphism $T$ between $\left[x_{j}\right]$ and $\left[y_{j}\right]$ with $T x_{j}=y_{j}$ for all $j$. Finally, we let Se denote the Banach space of all convergent series, i.e., all sequences $\left(c_{j}\right)$ with $\sum c_{j}$ convergent, under the norm $\left\|\left(c_{j}\right)\right\|_{S e}=\sup _{n}\left|\sum_{j=1}^{n} c_{j}\right|$; the summing basis refers to the unit-vector basis for $(\mathrm{Se})$, i.e., the sequence $\left(b_{j}\right)$ with $b_{j}(i)=\delta_{i j}$ for all $i$ and $j$. It is easily seen that Se is isomorphic to $c_{0}$; indeed, if $\left(e_{j}\right)$ denotes the standard (i.e., unit-vector basis) for $c_{0}$, then setting $b_{j}=\sum_{i=1}^{j} e_{i}$ for all $j$, $\left(b_{j}\right)$ is equivalent to the summing basis. 
For the remainder of this section, let $B$ denote a real or complex Banach space; we shall take $B$ to be infinite-dimensional, for the formulated results are trivial otherwise. We begin with several motivating corollaries and remarks.

We first observe that the two alternatives of Theorem 1.1 are mutually exclusive. Indeed, the summing basis is obviously not (s.s.), and it is evident that every convex block basis of the summing basis is equivalent to it; on the other hand, it is an easy permanence property of (s.s.)-sequences (as we show in Proposition 2.5) that every convex block basis of an (s.s.)-sequence is also (s.s.). Now suppose to the contrary, that $\left(f_{n}\right)$ is a non-trivial weak-Cauchy sequence, and we had $\left(g_{n}\right)$ and $\left(h_{n}\right)$ convex block bases of $\left(f_{n}\right)$ with $\left(g_{n}\right)$ an (s.s.)-sequence and $\left(h_{n}\right)$ equivalent to the summing basis.

It follows (since $\left(g_{n}\right)$ and $\left(h_{n}\right)$ converge weak* to the same element of $B^{* *}$ ) that $\left(g_{n}-h_{n}\right)$ is weakly null. But then there exist convex block bases $\left(\mathbf{g}_{n}\right)$ of $\left(g_{n}\right)$ and $\left(\mathbf{h}_{n}\right)$ of $\left(h_{n}\right)$ with $\left\|\mathbf{g}_{n}-\mathbf{h}_{n}\right\|<1 / 2^{n}$ for all $n$ (since there is a convex block basis of $\left(g_{n}-h_{n}\right)$ tending to zero in norm). But then a standard perturbation result yields that $\left(\mathbf{g}_{n}\right)$ and $\left(\mathbf{h}_{n}\right)$ are equivalent basic sequences, and hence $\left(\mathbf{g}_{n}\right)$ is an (s.s.)-sequence which is equivalent to the summing basis; this contradiction proves the assertion.

Now of course Theorem 1.0 (and in fact the author's original work in [R1]) yields immediately that $B$ contains no isomorph of $\ell^{1}$ if and only if every bounded sequence in $B$ has a weak-Cauchy subsequence. The next result yields the analogous characterization of spaces not containing $c_{0}$. It follows immediately from Theorem 1.1, since the summing basis spans $\mathrm{Se}$, a space isomorphic to $c_{0}$.

Corollary 1.2. B contains no isomorph of $c_{0}$ if and only if every non-trivial weak-Cauchy sequence in $B$ has an (s.s.)-subsequence.

Remark. Corollary 1.2 and known results yield the following "dual" characterization of Banach spaces containing $\ell^{1}: B$ contains no isomorph of $\ell^{1}$ if and only if, for every linear subspace $X$ of $B$, every non-trivial weak-Cauchy sequence in $X^{*}$ has an (s.s.)-subsequence. Since the summing basis has no (s.s.)-subsequence, one direction is completely trivial. For the other, suppose $X$ is a linear subspace of $B$ and there is a non-trivial weak-Cauchy sequence in $X^{*}$ with no (s.s.)-subsequence. Then $c_{0}$ embeds in $X^{*}$, by Corollary 1.2 , so $\ell^{1}$ embeds in $X$ by a result of Bessaga-Pełczyński [Bes-P].

In order to discuss the next corollary, we recall the following Banach space construct:

Definition 1.2(a). A sequence $\left(x_{j}\right)$ in $B$ is called (WUC) (Weakly Unconditionally Cauchy) if $\sum\left|b^{*}\left(x_{j}\right)\right|<\infty$ for all $b^{*} \in B^{*}$.

(In the literature, (WUC)-sequences are also termed (wus), for weakly unconditionally summing). Evidently if $\left(x_{j}\right)$ is (WUC), then setting $f_{n}=\sum_{j=1}^{n} x_{j}$ for all $n,\left(f_{n}\right)$ is weak-Cauchy. We crystallize this class of weak-Cauchy sequences as follows.

Definition 1.2(b). A sequence $\left(f_{n}\right)$ in $B$ is called (DUC) (for Difference (Weakly) Unconditionally Cauchy) if $\left(f_{n+1}-f_{n}\right)_{n=1}^{\infty}$ is (WUC). 
We now consider a notion introduced by A. Pełczyński [P1].

Definition 1.3. $B$ has property $(u)$ if for every weak-Cauchy sequence $\left(x_{j}\right)$ in $B$, there exists a (DUC)-sequence $\left(f_{j}\right)$ in $B$ so that $\left(x_{j}-f_{j}\right)_{j=1}^{\infty}$ is weakly null.

Of course it is trivial that, in the definition, we may restrict ourselves to non-trivial weak-Cauchy sequences.

The next result provides one of the main motivations for Theorem 1.1.

Corollary 1.3. If $B$ is non-reflexive and $X^{*}$ is weakly sequentially complete for all linear subspaces $X$ of $B$, then $c_{0}$ embeds in $B$; in fact, $B$ has property (u).

To see this, we use the following simple permanence property of (s.s.)sequences, proved in Section 2 (Proposition 2.4).

Proposition 1.4. Let $\left(b_{j}\right)$ be an (s.s.)-sequence in $B$, and $\left(b_{j}^{*}\right)$ the biorthogonal functionals for $\left(b_{j}\right)$ in $\left[b_{j}\right]^{*}$. Then $\left(\sum_{j=1}^{n} b_{j}^{*}\right)$ is a non-trivial weak-Cauchy sequence; hence $\left[b_{j}\right]^{*}$ is not weakly sequentially complete.

Proof of Corollary 1.3. The second assertion implies the first by general principles, but it's easier just to prove the claims in turn, directly. Now the hypotheses imply that $\ell^{1}$ doesn't embed in $B$, for after all $c_{0}$ embeds in $\left(\ell^{1}\right)^{*}=\ell^{\infty}$ and $c_{0}$ is not weakly sequentially complete. Since $B$ is non-reflexive, it follows by the $\ell^{1}$-Theorem (i.e., Theorem 1.0) that $B$ has a non-trivial weak-Cauchy sequence $\left(x_{j}\right)$. But $\left(x_{j}\right)$ cannot have an (s.s.)-subsequence by Proposition 1.4, so $\left(x_{j}\right)$ has a convex block basis equivalence to the summing basis by our main result, Theorem 1.1. Thus $c_{0}$ embeds in $B$. Again, if $\left(x_{j}\right)$ is a non-trivial weak-Cauchy sequence in $B$, then letting $\left(f_{j}\right)$ be a convex block basis equivalence to the summing basis, $\left(x_{j}-f_{j}\right)_{j=1}^{\infty}$ is weakly null, and of course $\left(f_{j}\right)$ is (DUC), so $B$ has property $(u)$.

The above reasoning, together with standard results, yields the following equivalence.

Corollary 1.5. Let $B$ be given. The following are equivalent.

(1) $X^{*}$ is weakly sequentially complete for all linear subspaces $X$ of $B$.

(2) $B$ has property $(u)$ and $\ell^{1}$ does not embed in $B$.

Proof. (1) $\Rightarrow(2)$ follows immediately from Corollary 1.3.

$(2) \Rightarrow(1)$. Suppose $X$ is a linear subspace of $B$, yet $X^{*}$ is not weakly sequentially complete. Then as we show in Section 2 (cf. Proposition 2.6), since $\ell^{1}$ does not embed in $X, X$ contains an (s.s.)-sequence $\left(x_{j}\right)$. Suppose there were a (DUC)-sequence $\left(f_{j}\right)$ with $\left(x_{j}-f_{j}\right)_{j=1}^{\infty}$ weakly null. But now it follows that $\left(f_{j}\right)$ has a subsequence $\left(g_{j}\right)$ equivalent to the summing basis. (See Section 3, Corollary 3.3.) But then $\left(x_{j}-g_{j}\right)$ is again weakly null, so as in the argument proving the mutual exclusivity of the alternatives of Theorem 1.1, we finally obtain a convex block basis $\left(y_{j}\right)$ of $\left(x_{j}\right)$ which is equivalent to the summing basis and (s.s.), a contradiction. 
Remark. A crystallization of known arguments yields the fact (proved here in Proposition 3.2 for the sake of completeness): If $\left(f_{j}\right)$ is a DUC sequence, then so is every convex block basis $\left(y_{j}\right)$ of $\left(f_{j}\right)$. Thus we see immediately the standard result that property $(u)$ is hereditary. On the other hand, it follows by the results in [P2] and the $\ell^{1}$-Theorem that if $X$ has property $(u)$ and $\ell^{1}$ does not embed in $X$, then $X^{*}$ is weakly sequentially complete. Thus $(2) \Rightarrow(1)$ of 1.5 may instead be proved using these old results. We also note the various properties $(V)$ and $\left(V^{*}\right)$ introduced in [P2], where it is shown that for a particular space $X$,

$$
(u) \Rightarrow(V) \Rightarrow\left(V^{*}\right) \Rightarrow X^{*} \text { is weakly sequentially complete. }
$$

Thus we obtain that $B$ has any of these properties hereditarily if and only if $B$ satisfies (2) of Corollary 1.5. In particular, this answers a question posed in [R4] in the affirmative (cf. the end of Section 3 of [R4]).

The criterion for embedding $c_{0}$ in a given $B$, given by Corollary 1.3 , involves "checking" all the linear subspaces $X$ of $B$. The next result gives a stronger criterion, for fewer subspaces need to be checked.

Corollary 1.6. Suppose $B$ is a non-reflexive space with a basis $\left(b_{j}\right)$, so that every block basis of $\left(b_{j}\right)$ spans a space with weakly sequentially complete dual. Then $c_{0}$ embeds in $B$.

Proof. Suppose, to the contrary, that $c_{0}$ does not embed in $B$. Then we have that

$$
\left(b_{j}\right) \text { is shrinking. }
$$

That is, $\left[b_{j}^{*}\right]=B^{*}$, where $\left(b_{j}^{*}\right)$ denotes the biorthogonal functionals to $\left(b_{j}\right)$. An equivalent formulation: every normalized block basis $\left(u_{j}\right)$ of $\left(b_{j}\right)$ is weakly null. So, suppose we had $\left(u_{j}\right)$ a normalized block basis which is not weakly null. Now standard results show that $\left(u_{j}\right)$ has a subsequence $\left(u_{j}^{\prime}\right)$ with no further weakly convergent subsequences. Then $\left(u_{j}^{\prime}\right)$ could not have an $\ell^{1}$ subsequence $\left(u_{j}^{\prime \prime}\right)$, for then $\left[u_{j}^{\prime \prime}\right]^{*}$ would not be weakly sequentially complete. Thus the $\ell^{1}$-Theorem (Theorem 1.0) yields that $\left(u_{j}^{\prime}\right)$ has a non-trivial weakCauchy sequence $\left(v_{j}\right)$. But in turn, Corollary 1.2 implies $\left(v_{j}\right)$ has an (s.s.)subsequence $\left(v_{j}^{\prime}\right)$, which contradicts the hypotheses by Proposition 1.4. Thus (1) is established.

Now it follows that $\left(b_{j}\right)$ cannot be boundedly complete, or else $B$ would be reflexive. That is, we may choose scalars $c_{1}, c_{2}, \ldots$ so that

$$
\sup _{n}\left\|\sum_{j=1}^{n} c_{j} b_{j}\right\|<\infty \text { but } \sum c_{j} b_{j} \text { does not converge. }
$$

Next let $f_{n}=\sum_{j=1}^{n} c_{j} b_{j}$ for all $n$. Of course $\left(f_{n}\right)$ is a bounded sequence; we also have that if $n_{1}<n_{2}<\cdots$ are given, then

$$
\left[f_{n_{i}}\right]=\left[u_{i}\right] \text { for a certain block basis }\left(u_{i}\right) \text { of }\left(b_{j}\right) \text {; }
$$


namely let $u_{1}=f_{n_{1}}$ and $u_{j}=f_{n_{j}}-f_{n_{j-1}}$ for $j>1$. But again $\left(f_{n}\right)$ can thus have no $\ell^{1}$-subsequence, so by Corollary 1.2, $\left(f_{n}\right)$ must have an (s.s.)subsequence $\left(f_{n_{i}}\right)_{i=1}^{\infty}$ which contradicts the hypotheses by (3) and Proposition 1.4.

Remark. Suppose $B$ satisfies the hypotheses of Corollary 1.6. Does $B$ have property $(u)$ ? I suspect the answer is no, in general.

The argument for Corollary 1.6, when phrased directly, has a natural interpretation in terms of (s.s.)-sequences, and a fundamental companion notion.

Definition 1.4. A basic sequence $\left(e_{j}\right)$ in a Banach space is called (c.c.) (for coefficient converging) if

(i) $\left(\sum_{j=1}^{n} e_{j}\right)$ is a weak-Cauchy sequence and

(ii) for any scalars $\left(c_{j}\right)$, if $\sup _{n}\left\|\sum_{j=1}^{n} c_{j} e_{j}\right\|<\infty$, then the sequence $\left(c_{j}\right)$ converges.

Now we prove in Section 2 (cf. Proposition 2.3) that if $\left(b_{j}\right)$ is an (s.s.)sequence, then its difference sequence $\left(e_{j}\right)_{j=1}^{\infty}$ is (c.c.) (where $e_{j}=b_{j}-b_{j-1}$ for $\left.j>1, e_{1}=b_{1}\right)$; conversely if $\left(e_{j}\right)$ is a (c.c.)-sequence, then setting $b_{j}=$ $\sum_{i=1}^{j} e_{i}$ for all $j,\left(b_{j}\right)$ is an (s.s.)-sequence. The argument for Corollary 1.6 then yields the following result. Suppose neither $c_{0}$ nor $\ell^{1}$ embeds in $B$, and $\left(b_{j}\right)$ is a given basic sequence in $B$. If $\left(b_{j}\right)$ is not shrinking, $\left(b_{j}\right)$ has an (s.s.) block basis; if $\left(b_{j}\right)$ is not boundedly complete, $\left(b_{j}\right)$ has a (c.c.) block basis.

Remark. The "hereditary" hypotheses in Corollaries 1.3, 1.6, and in the result formulated in the Remark following Corollary 1.2, are crucial. Indeed, Bourgain-Delbaen [Bo-De] have constructed a Banach space $X$ so that $c_{0}$ does not embed in $X$, yet $X^{*}$ is isomorphic to $\ell^{1}$, so of course $X$ is non-reflexive and $X^{*}$ is weakly sequentially complete.

Concerning the remark after Corollary 1.2 , of course $\ell^{1}$ embeds in $C[0,1]$, yet $(C[0,1])^{*}$ is weakly sequentially complete, so it has no non-trivial weakCauchy sequences. A more interesting example: there is constructed in [BoDe] a space $Y$ with the Schur property (i.e., every weak-Cauchy sequence in $Y$ is norm-convergent), with $Y^{*}$ isomorphic to $C[0,1]^{*}$. Thus $Y^{*}$ is again weakly sequentially complete, and (by Theorem 1.0) $\ell^{1}$ embeds in every infinite-dimensional subspace of $Y$.

As in the case of the $\ell^{1}$-Theorem, the $c_{0}$-theorem is proved by reducing to a "classical real variables" setting. The following concept is crucial.

Definition 1.5. Let $K$ be a compact metric space and $f: K \rightarrow \mathbb{C}$ a given function. $f$ is a (complex) difference of bounded semi-continuous functions if there exist continuous complex-valued continuous functions $\varphi_{1}, \varphi_{2}, \ldots$ on $K$ with $\sup _{k \in K} \sum\left|\varphi_{j}(k)\right|<\infty$ and $f=\sum \varphi_{j}$ point-wise. We let $D(K)$ denote the family of all such functions; we also let $N D(K)$ denote the set of all bounded functions $f$ on $K$ which don't belong to $D(K)$.

The reason for the terminology is as follows: let $f: K \rightarrow[-\infty, \infty]$ be an 
extended real-valued function. $f$ is called upper semi-continuous if $f(x)=$ $\overline{\lim }_{y \rightarrow x} f(y)$ for all $x \in K ; f$ is called lower semi-continuous if $f(x)=$ $\varliminf_{y \rightarrow x} f(y)$ for all $x \in K$. (Following Bourbaki, we use non-exclusive lim sups and $\lim$ infs; thus $\varlimsup_{y \rightarrow x} f(y)=\inf _{x \in U} \sup _{y \in U} f(y)$, the inf over all open neighborhoods of $x$; equivalently, $\varlimsup_{y \rightarrow x} f(y)=\max \left\{L \in[-\infty, \infty]: \exists x_{n} \rightarrow\right.$ $x, f\left(x_{n}\right) \rightarrow L$ as $\left.n \rightarrow \infty\right\}, f$ is called semi-continuous if it is either upper or lower semi-continuous. It then follows from results of Baire that $f \in D(K)$ if and only if there are bounded lower semi-continuous functions $u_{1}, \ldots, u_{4}$ on $K$ so that $f=\left(u_{1}-u_{2}\right)+i\left(u_{3}-u_{4}\right)$.

Evidently, if $g \in D(K)$, then $g \in B_{1}(K)$, the first Baire class of bounded functions on $K$, i.e., the set of all functions $f$ on $K$ so that there exists a uniformily bounded sequence $\left(f_{n}\right)$ in $C(K)$ with $f_{n} \rightarrow f$ point-wise. The following result gives the fundamental connection between $f$ and the Banach space structure of this sequence $\left(f_{n}\right)$. The result follows from refinements of arguments in [Bes-P] and is explicitly stated in Corollary 3.5 of [HOR].

Proposition 1.7. Let $K$ be a compact metric space, $f: K \rightarrow \mathbb{C}$ discontinuous, and $\left(f_{n}\right)$ a uniformly bounded sequence of continuous functions on $K$ with $f_{n} \rightarrow f$ point-wise. Regarding $f_{1}, f_{2}, \ldots$ as lying in the Banach space $C(K)$, then $f$ is in $D(K)$ if and only if $\left(f_{n}\right)$ has a convex block basis equivalent to the summing basis.

For the sake of completeness, we give the proof in Section 3 (following Corollary 3.3). Now the $c_{0}$-theorem follows immediately from 1.7 and the following "real-variables" result.

Theorem 1.8. Let $K$ be a compact metric space, $f$ a complex-valued function on $K$, and $\left(f_{n}\right)$ a uniformly bounded sequence of complex-valued continuous functions on $K$ with $f_{n} \rightarrow f$ point-wise. Then if $f$ is not in $D(K),\left(f_{n}\right)$ has an (s.s.)-subsequence.

To deduce Theorem 1.1, let $X$ be a separable Banach space, and let $K$ denote the unit ball of $X^{*}$ endowed with the weak*-topology. Let us denote by $X_{D}^{* *}$ (resp. $X_{\mathbf{B}_{1}}^{* *}$ ) the set of all $x^{* *} \in X^{* *}$ with $x^{* *} \mid K \in D(K)$ (resp. $x^{* *} \mid K \in$ $\left.B_{1}(K)\right)$. Now suppose $\left(x_{n}\right)$ is a non-trivial weak-Cauchy sequence in $X$, and let $x^{* *}$ denote its weak*-limit in $X^{* *}$; also let $\chi: X \rightarrow X^{* *}$ denote the canonical embedding. If $x^{* *} \in X_{D}^{* *}$, then Proposition 1.7 yields that $\left(\chi x_{n}\right) \mid K$ has a convex block basis equivalence to the summing basis in $C(K)$, so of course $\left(x_{n}\right)$ has exactly the same property in $X$. Again if $x^{* *} \notin X_{D}^{* *}$, Theorem 1.8 yields that $\left(\chi x_{n}\right) \mid K$ and hence $\left(x_{n}\right)$ has an (s.s.)-subsequence.

We finally note a consequence of our results, formulated in terms of the classes $X_{D}^{* *}$ and $X_{\mathbf{B}_{1}}^{* *}$, for $X$ separable. Standard results (cf. [OR]) yield that $x^{* *} \in X_{\mathbf{B}_{1}}^{* *} \backslash X$ if and only if there is a non-trivial weak-Cauchy sequence $\left(x_{n}\right)$

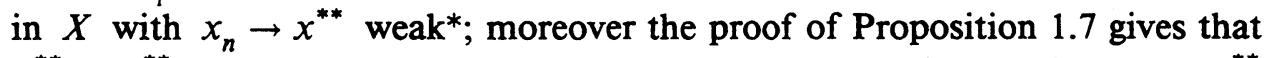
$x^{* *} \in X_{D}^{* *}$ if and only if there is a DUC sequence $\left(x_{n}\right)$ in $X$ with $x_{n} \rightarrow x^{* *}$ weak*. It thus follows that $X$ has property $(u)$ if and only if $X_{\mathbf{B}_{1}}^{* *}=X_{D}^{* *}$. A result of $\mathrm{E}$. Odell and the author [OR] asserts that $\ell^{1} \nrightarrow X$ if and only if 
$X^{* *}=X_{\mathbf{B}_{1}}^{* *}$. We may then combine the above and Corollary 1.3 to obtain the following result (where we let $X_{N D}^{* *}=X^{* *} \backslash X_{D}^{* *}$ ).

Corollary 1.9. Let $X$ be a separable Banach space. The following are equivalent.

(1) Neither $c_{0}$ nor $\ell^{1}$ embeds in $X$.

(2) $X_{\mathbf{B}_{1}}^{* *} \cap X_{N D}^{* *}=X^{* *} \backslash X$.

(3) For all non-reflexive linear subspaces $Y$ of $X$, there exists a linear subspace $Z$ of $Y$ so that neither $Z$ nor $Z^{*}$ is weakly sequentially complete.

Remark. Under rather strong hypotheses, one can obtain sequences which are "better behaved" than (s.s.)-sequences. Thus in [R6], it is proved that every non-trivial weak-Cauchy sequence in a Banach space with the PCP (the point of continuity property) has a boundedly complete subsequence. By Proposition 2.2 below, this subsequence can be chosen to be $(s)$ as defined in Section 2. In particular, this holds in any separable Banach space isomorphic to a dual space. Of course boundedly complete (s)-sequences are (s.s.); but this considerably stronger property seems rather rare, among general Banach spaces. For example, W.T. Gowers [Go] has recently constructed an infinite-dimensional Banach space $X$ which contains no subspace isomorphic to $c_{0}$ and no infinitedimensional subspace isomorphic to a dual space. It follows from our results (i.e., Theorems 1.0 and 1.1) that then every infinite-dimensional subspace of $X$ contains an (s.s.)-sequence, yet $X$ has no boundedly-complete basic sequences.

We now indicate the organization of the remaining sections of this article. Section 2 deals with permanence properties of (s.s.)-sequences. (Throughout, results are numbered consecutively in each section, with definitions being separately numbered.) We show in Proposition 2.3 that a sequence is (s.s.) if and only if its difference sequence is (c.c.); and in Proposition 2.4 that a basic sequence is (s.s.) if and only if its sequence of biorthogonal functionals is (c.c.). Proposition 1.4 is of course an immediate consequence of 2.4. Proposition 2.7 yields the result that a sequence is (s.s.) if and only if every proper subsequence of its difference sequence is semi-boundedly complete. (A seminormalized basic sequence $\left(x_{j}\right)$ in a Banach space is called semi-boundedly complete if whenever $\sup _{n}\left\|\sum_{j=1}^{n} c_{j} x_{j}\right\|<\infty$, then $c_{j} \rightarrow 0$; this is equivalent to the assertion that $x_{j}^{*} \rightarrow 0$ weakly, where $\left(x_{j}^{*}\right)$ is biorthogonal to $\left(x_{j}\right)$.) Our characterization of spaces containing $c_{0}$ is thus related to the one of J. Elton [E]: If $\left(x_{j}\right)$ is a normalized weakly null sequence in a Banach space with no subsequence equivalent to the $c_{0}$-basis, then $\left(x_{j}\right)$ has a semi-boundedly complete subsequence.

We introduce an " $\varepsilon$-version" of (c.c.)-sequences in Definition 2.4, and show in Lemma 2.8 that a non-trivial weak-Cauchy sequence has an (s.s.)-subsequence provided for every $\varepsilon>0$, every subsequence has a further subsequence where differences are $\varepsilon-$ (c.c.). This result is used directly in the proof of Theorem 1.1.

In Section 3, we first deal with some permanence properties of DUC sequences. Thus we show in Proposition 3.1 that a sequence is equivalent to the summing basis if and only if it is an (s)-sequence which is DUC. We show that DUC-sequences are preserved by taking convex block bases in Proposition 3.2, and then (after Corollary 3.3) give the proof of Proposition 1.7. 
The rest of Section 3, as well as most of Section 4, may be read independently of the rest of the paper and in fact of Banach space theory. The results here lie more in the setting of descriptive set theory and classical real variables, rather than Banach spaces, although the latter gives their primary motivation. We first take up the development of the intrinsic invariants of a difference of bounded semi-continuous functions, introducing in Definition 3.1 the transfinite oscillations osc $_{\alpha} f$ of a complex-valued function defined on a separable metric space $K$. We prove in Theorem 3.5 that if $f: K \rightarrow \mathbb{C}$ is a bounded function, then $f$ is in $D(K)$ if and only if $\operatorname{osc}_{\alpha} f$ is a bounded function for all $\alpha$. Moreover, if $f$ is real-valued and this happens, there is an $\alpha$ so that $\operatorname{osc}_{\alpha} f=$ osc $_{a+1} f$ and then

$$
\|f\|_{D}=\left\||f|+\operatorname{osc}_{\alpha} f\right\|_{\infty}
$$

where

$\|f\|_{D}=\inf \left\{\sup _{t} \sum\left|\varphi_{j}(t)\right|: f=\sum \varphi_{j}\right.$ point-wise, the $\varphi_{j}$ 's continuous on $\left.K\right\}$.

A surprising consequence of our work here is that this infimum is attained.

The transfinite oscillations osc $f$ are related to earlier invariants introduced by A.S. Kechris and A. Louveau [KL], termed by us here the positive oscillations $v_{a}(f)$ of a real-valued function $f$ (see Definition 3.2). We show the natural inequalities connecting these with the transfinite oscillations in Proposition 3.8; for the Banach space context of interest here, these invariants have essentially the same growth (see the Remark preceding Lemma 3.9). The fact that $f$ is in $D(K)$ if and only if osc $f$ is bounded for all $\alpha$ already follows from the earlier work in $[\mathrm{KL}]$. The transfinite oscillations seem to us more appropriate to Banach space structure than the transfinite positive oscillations, and these invariants are used to obtain further structure theorems for differences of semicontinuous functions and related Baire-1 classes in [R5]. Nevertheless, it turns out to be more convenient to use the $v_{\alpha}(f)$ 's to prove our main result, reduced to Theorem 1.8 above, and Section 3 concludes with a technical result relating $v_{\alpha}(f)$ and $v_{\alpha+1}(f)$, used in this proof (Lemma 3.9).

Sections 2 and 3 thus set up the needed invariants (with complementary results), and Section 4 is then devoted to the proof of our main result. The heart of the matter is contained in the "real-variables" result, Theorem 4.1. This result shows that if $f_{j} \rightarrow f$ point-wise on $K$ a separable metric space with the $f_{j}$ 's uniformly bounded complex-valued functions, $\alpha$ is a countable ordinal, and $0<v_{\alpha}(\operatorname{Re} f)(x)<\infty$ for some $x \in X$, then there is a subsequence $\left(b_{j}\right)$ of the $f_{j}$ 's so that all further subsequences "witness" the quantity $v_{\alpha}(\operatorname{Re} f)(x) \stackrel{\text { df }}{=}$ $\lambda$. The quantitative information of this theorem then yields that if $\varepsilon>0$ is given and $\lambda$ is large enough, then every subsequence of $\left(b_{j}\right)$ has its difference sequence $\varepsilon-$ (c.c.). Thus to prove Theorem 1.8, we let $\varepsilon>0$ be given. Then we choose $\alpha$ so that $v_{\alpha}(\operatorname{Re} f)$ is a bounded function but $\left\|v_{\alpha}(\operatorname{Re} f)\right\|_{\infty}>2 / \varepsilon$. Now Theorem 4.1 allows us to show that $\left(f_{j}\right)$ satisfies the hypotheses of Lemma 2.8, whence $\left(f_{j}\right)$ has an (s.s.)-subsequence. 
Theorem 4.1 is formulated directly in terms of difference sequences; we "reformulate" the needed information concerning the behavior on appropriate subsequences of $\left(f_{j}\right)$ as Theorem 4.2. We then give the easy demonstration that Theorem 4.2 implies Theorem 4.1, and the balance of Section 4 is devoted to the rather delicate proof of Theorem 4.2 itself. This argument is accomplished by transfinite induction; the entire proof follows very quickly from the " $\alpha$ to $\alpha+1$ " step. A rather surprising feature of the argument is that using only the $\alpha$-information, but not how it is obtained (i.e., its "history"), and a careful discussion giving the " $\alpha=1$ " case (Lemma 4.3), we obtain the $\alpha+1$-st case. The actual subsequences are constructed in Sub-Lemma 1 for the " $\alpha=1$ " case, and in Sub-Lemma 2 for the " $\alpha$ to $\alpha+1$ " case.

The results given here were presented in a topics course at The University of Texas at Austin in 1991-1992. The formulations and discoveries were then very much in the trial and error stage, with the main theorem being established only in April. I am most grateful to the participants in this course for their patience and helpful comments concerning this work.

\section{Permanence PROPERTies of (s.S.)-SeQuences}

We first define a notion weaker than that of (s.s.)-sequences (this concept appears in [HOR], without the terminology).

Definition 2.1. A sequence $\left(b_{j}\right)$ in a Banach space is called an (s)-sequence ("(s)" is for "summing") if $\left(b_{j}\right)$ is a weak-Cauchy basic sequence which dominates the summing basis.

It is evident that if $\left(b_{j}\right)$ is an (s)-sequence, then $\left(b_{j}\right)$ is non-trivial weakCauchy. Now in fact standard arguments give that every non-trivial weak-Cauchy sequence has an (s)-subsequence (cf. [HOR]); since certain estimates in the argument are needed for our main result, we sketch the proof below. We also note that if $\left(b_{j}\right)$ is weak-Cauchy and basic, then $\left(b_{j}\right)$ is $(\mathbf{s})$ if and only if whenever $\left(c_{j}\right)$ is a sequence of scalars with $\sum c_{j} b_{j}$ convergent, then $\sum c_{j}$ converges. Thus trivially (s.s.)-sequences are (s)-sequences. Now it follows that if $\left(b_{j}\right)$ is (s), there is a unique $\mathbf{s} \in\left[b_{j}\right]^{*}$ with $\mathbf{s}\left(\sum c_{j} b_{j}\right)=\sum c_{j}$ for all $x \in\left[b_{j}\right]$ with $x=\sum c_{j} b_{j}$. We refer to $\mathbf{s}$ as the summing functional.

A natural companion notion to (s)-sequences is the following one. (A sequence $\left(x_{j}\right)$ in a Banach space is called semi-normalized (resp. normalized) if $\sup _{j}\left\|x_{j}\right\|<\infty$ and $\inf _{j}\left\|x_{j}\right\|>0$ (resp. $\left\|x_{j}\right\|=1$ for all $\left.j\right)$.)

Definition 2.2. A basic sequence $\left(e_{j}\right)$ in a Banach space is called a (c)-sequence (“(c)" is for "convergent") if $\left(e_{j}\right)$ is a semi-normalized basic sequence so that $\left(\sum_{j=1}^{n} e_{j}\right)_{n=1}^{\infty}$ is weak-Cauchy.

We next give a simple relationship between these notions. (Throughout, given sequences $\left(b_{j}\right)$ and $\left(e_{j}\right)$ in a Banach space, $\left(e_{j}\right)$ is called the difference sequence of $\left(b_{j}\right)$ if $e_{1}=b_{1}$ and $e_{j}=b_{j}-b_{j-1}$ for all $j>1$. Also, if $\left(b_{j}\right)$ is a basic sequence, then $\left(b_{j}^{*}\right)$ denotes its sequence of biorthogonal functionals in $\left[b_{j}\right]^{*}$; 
i.e., $b_{j}^{*}\left(b_{i}\right)=\delta_{i j}$ for all $i$ and $j$. It is a standard result that then $\left(b_{j}^{*}\right)$ is also a basic sequence.)

Proposition 2.1. Let $\left(b_{j}\right)$ be a given sequence in a Banach space, and $\left(e_{j}\right)$ be its difference sequence. Then $\left(b_{j}\right)$ is (s) if and only if $\left(e_{j}\right)$ is (c).

Remark. Of course it's then trivial that $\left(b_{j}\right)$ and $\left(e_{j}\right)$ are both bases for $\left[b_{j}\right]$. It is also immediate that every (c.c.)-sequence is a (c)-sequence.

Proof. Suppose first that $\left(b_{j}\right)$ is an (s)-sequence, and let $\left(P_{k}\right)$ be its basis projections. That is, for all $k, P_{k}:\left[b_{j}\right] \rightarrow\left[b_{j}\right]$ is defined by $P_{k} x=\sum_{j=1}^{k} c_{j} b_{j}$ if $x=\sum_{j=1}^{\infty} c_{j} b_{j}$. Also, let $\lambda$ be the basis-constant of $\left(b_{j}\right)$; that is, $\lambda=\sup _{k}\left\|P_{k}\right\|$. Finally, let $\mathbf{s}$ be the summing functional on $\left[b_{j}\right]$.

We then have that defining $e_{n}^{*}$ for all $n$ by

$$
e_{n}^{*}=\mathbf{s}-\sum_{i=1}^{n-1} b_{i}^{*} \text { for } n>1 \text { and } e_{1}^{*}=\mathbf{s}
$$

then $\left(e_{n}^{*}\right)$ is biorthogonal to $\left(e_{j}\right)$. Moreover since for all $n, \sum_{i=1}^{n} b_{i}^{*}=\mathbf{s} P_{n}$, it follows that

$$
\sup _{n}\left\|e_{n}^{*}\right\| \leq\|\mathbf{s}\|(1+\lambda) .
$$

Finally, to see that $\left(e_{j}\right)$ is basic, since $\left(e_{j}\right)$ is trivially linearly independent, it suffices to estimate the norms of its basis-projections on its linear span; that is, let $X_{0}$ denote the linear span of $\left(e_{j}\right)$ and for each $k$, set $Q_{k}\left(\sum c_{j} e_{j}\right)=$ $\sum_{j=1}^{k} c_{j} e_{j}$ for all $\sum c_{j} e_{j}$ in $X_{0}$; we need only estimate $\sup _{k}\left\|Q_{k}\right\|_{X_{0}}$.

Now let $k<n$ and $x=\sum_{j=1}^{n} c_{j} e_{j}$. Then

$$
\begin{aligned}
\sum_{j=1}^{n} c_{j} e_{j} & =c_{1} b_{1}+c_{2}\left(b_{2}-b_{1}\right)+\cdots+c_{n}\left(b_{n}-b_{n-1}\right) \\
& =\left(c_{1}-c_{2}\right) b_{1}+\cdots+\left(c_{n-1}-c_{n}\right) b_{n-1}+c_{n} b_{n} .
\end{aligned}
$$

Hence

$$
\sum_{j=1}^{k} c_{j} e_{j}=P_{k-1} x+e_{k}^{*}(x) b_{k} \quad\left(\text { where we set } P_{0}=0\right) .
$$

That is, we have proved

$$
Q_{k}=P_{k-1}+e_{k}^{*} \otimes b_{k}
$$

(where for $X$ a Banach space, $x \in X, x^{*} \in X^{*}, x^{*} \otimes x$ denotes the rank-one operator $\left(x^{*} \otimes x\right)(y)=x^{*}(y) x$ for all $\left.y \in X\right)$. Evidently we thus have that $\sup _{k}\left\|Q_{k}\right\|<\infty$; in fact

$$
\sup _{k}\left\|Q_{k}\right\| \leq \lambda+(1+\lambda)\|\mathbf{s}\| \sup _{k}\left\|b_{k}\right\| .
$$

Since $\left(b_{k}\right)$ is non-trivial weak-Cauchy, it is semi-normalized; and because it's a basic sequence, its difference sequence $\left(e_{j}\right)$ is also semi-normalized. Of course 
since $\sum_{j=1}^{n} e_{j}=b_{n}$ for all $n,\left(\sum_{j=1}^{n} e_{j}\right)$ is weak-Cauchy, and hence $\left(e_{j}\right)$ is a (c)-sequence.

Conversely, suppose $\left(e_{j}\right)$ is a $(c)$-sequence. Then trivially $\left(b_{j}\right)$ is weakCauchy. Since $\left(e_{j}\right)$ is semi-normalized, $\left(e_{k}^{*}\right)$ is bounded. But then we may use (7) to obtain that if $\left(P_{k}\right)$ is the sequence of basis projections of $\left(b_{j}\right)$ on $X_{0}$, then, for all $k$,

$$
P_{k}=Q_{k+1}-e_{k+1}^{*} \otimes b_{k+1}
$$

hence $\sup _{k}\left\|P_{k}\right\| \leq \sup _{k}\left\|Q_{k}\right\|+\sup _{k}\left\|e_{k}^{*}\right\|\left\|b_{k}\right\|<\infty$.

Thus $\left(b_{k}\right)$ is a basic sequence; since $e_{1}^{*}\left(b_{j}\right)=1$ for all $j, e_{1}^{*}$ is indeed the summing functional on $\left[b_{j}\right]$, whence $\left(b_{j}\right)$ is $(s)$.

Remark. Let $\left(b_{j}\right),\left(e_{j}\right)$ be given sequences in a Banach space. Say that $\left(b_{j}\right)$ is wide- $(\mathbf{s})$ if $\left(b_{j}\right)$ is a semi-normalized basic sequence which dominates the summing basis. Say that $\left(e_{j}\right)$ is wide- $(\mathrm{c})$ if $\left(e_{j}\right)$ is a semi-normalized basic sequence so that $\sup _{n}\left\|\sum_{j=1}^{n} e_{j}\right\|<\infty$. Then the above proof yields immediately that $\left(b_{j}\right)$ is wide- $(\mathbf{s})$ if and only if its difference sequence $\left(e_{j}\right)$ is wide- $(\mathrm{c})$. Thus in particular, if $\left(b_{j}\right)$ is a semi-normalized basic sequence, then its difference sequence $\left(e_{j}\right)$ is basic if and only if $\left(b_{j}\right)$ is wide- $(\mathbf{s})$.

We now refine a classical argument to obtain the universality of (s)-sequences in non-weakly sequentially complete spaces.

Proposition 2.2. Let $\left(x_{j}\right)$ be a non-trivial weak-Cauchy sequence in a Banach space. Then $\left(x_{j}\right)$ has an (s)-subsequence.

Remark. Of course it thus follows from the $\ell^{1}$-Theorem that every wide-(s) sequence has a subsequence which is either an (s)-sequence or an $\ell^{1}$-sequence (i.e., equivalent to the $\ell^{1}$-basis). We shall see below, however, that it is nevertheless natural to introduce the wide-notions of (s)- and (c)-sequences.

We sketch the proof of 2.2. Recall that for $X$ a Banach space and $Y$ a linear subspace of $X^{*}, Y$ is said to isomorphically norm $X$ if there is a constant $0<\eta$ so that

$$
\eta\|x\| \leq \sup _{y \in B a Y}|y(x)| \text { for all } x \in X .
$$

We also say $Y \quad \eta$-norms $X$ if (10) holds. Of course $\eta \leq 1$. In case $\eta=1$ in (10), we say $Y$ isometrically norms $X$. We use the following two standard results without proof. (A sequence $\left(b_{j}\right)$ is called $\lambda$-basic if it is basic with basis constant at most $\lambda$.)

Lemma 1. Let $X$ be a Banach space, $\left(x_{j}\right)$ a semi-normalized sequence in $X$, and $Y$ an isomorphically norming subspace of $X^{*}$ so that $y\left(x_{j}\right) \rightarrow 0$ as $j \rightarrow \infty$ for all $y \in Y$. Then $\left(x_{j}\right)$ has a basic subsequence. In fact, if $Y$-norms $X$, then, given $0<\varepsilon<\eta,\left(x_{j}\right)$ has a $\frac{1}{\eta-\varepsilon}$-basic subsequence.

Lemma 2. Let $X$ be a Banach space, $G \in X^{* *} \sim X$. Then $G^{\perp}$ isomorphically norms $X$, where $G^{\perp}=\left\{x^{*} \in X^{*}: G\left(x^{*}\right)=0\right\}$. 
Proof of Proposition 2.2. Let $\left(x_{j}\right)$ be a non-trivial weak-Cauchy sequence in $X$, and define $G \in X^{* *}$ by $G(f)=\lim _{j} f\left(x_{j}\right)$ for all $f \in X^{*}$. Then $G \in X^{* *} \sim X$ since $\left(x_{j}\right)$ is non-trivial; hence $G^{\perp}$ isomorphically norms $X$ by Lemma 2, so $\left(x_{j}\right)$ has a basic subsequence $\left(y_{j}\right)$ by Lemma 1 . Now choose $f \in X^{*}$ with $G(f)=1$. Hence $f\left(y_{j}\right) \rightarrow 1$ as $j \rightarrow \infty$. Finally, given $\tau>0$, choose $\left(b_{j}\right)$ a subsequence of $\left(y_{j}\right)$ with

$$
\left|1-f\left(b_{j}\right)\right|<\frac{\tau}{2^{j}} \text { for all } j .
$$

To see that $\left(b_{j}\right)$ is an (s)-sequence, we need only show that there is a $\beta<\infty$ so that

$$
\left|\sum_{j=1}^{n} c_{j}\right| \leq \beta\left\|\sum_{j=1}^{n} c_{j} b_{j}\right\| \text { for all } n \text { and scalars } c_{1}, \ldots, c_{n} .
$$

(It then follows that the summing functional $\mathbf{s}$ is well-defined with $\|\mathbf{s}\| \leq \beta$.) But given scalars $c_{1}, \ldots, c_{n}$ and setting $x=\sum_{j=1}^{n} c_{j} b_{j}$, we have that

$$
\begin{aligned}
\left|\sum_{j=1}^{n} c_{j}\right| & =\left|\sum_{j=1}^{n} c_{j} f\left(x_{j}\right)+\sum_{j=1}^{n} c_{j}\left(1-f\left(x_{j}\right)\right)\right| \\
& \leq\|f\|\|x\|+\sup _{j} \tau\left\|y_{j}^{*}\right\|\|x\| .
\end{aligned}
$$

Thus (14) holds with $\beta=\|f\|+\tau \sup _{j}\left\|y_{j}^{*}\right\|$. This completes the proof of Proposition 2.2.

Remarks. 1. The above argument yields the following quantitative information: if $x_{j} \rightarrow G$ weak ${ }^{*}$ and $\delta=\operatorname{dist}\left(\frac{G}{\|G\|}, X\right)$ then given $\varepsilon>0,\left(x_{j}\right)$ has an (s)subsequence $\left(b_{j}\right)$ with basis constant at most $\frac{1+\delta}{\delta}+\varepsilon$ and summing functional of norm at most $\frac{1}{\|G\|}+\varepsilon$.

2. Say that a sequence $\left(x_{j}\right)$ in a Banach space is (wcb) (for weak-Cauchy basic) if $\left(x_{j}\right)$ is a basic sequence and a non-trivial weak-Cauchy sequence. Now it is easily seen that if $\left(x_{j}\right)$ is (wcb), $c \neq 0$, and $\left(c_{j}\right)$ is a sequence of nonzero scalars with $c_{j} \rightarrow c$, then $\left(c_{j} x_{j}\right)$ is also (wcb). It also follows that if $\left(x_{j}\right)$ is $(w c b)$, then there exists such a sequence $\left(c_{j}\right)$ with $\left(c_{j} x_{j}\right)$ an (s)-sequence. Thus from the point of view of basic-sequence permanence properties, (wcb) sequences appear as the more natural objects. However because of the essential property given by Proposition 2.1, we always pass to subsequences to obtain (s)-sequences. We note also that (wcb) sequences need not be (s); indeed, if $\left(b_{j}\right)$ is the summing basis itself and $c_{n}^{-1}=1+(-1)^{n} / \log (n+1)$ for all $n$, then $\left(c_{n} b_{n}\right)$ is (wcb) but not (s).

We pass now to the elementary permanence properties of (s.s.)- and (c.c.)sequences.

Proposition 2.3. Let $\left(b_{j}\right)$ be a given sequence in a Banach space, and $\left(e_{j}\right)$ be its difference sequence. Then $\left(b_{j}\right)$ is (s.s.) if and only if $\left(\boldsymbol{e}_{j}\right)$ is (c.c.). 
Proof. This follows simply from Proposition 2.1 and its proof. Suppose first that $\left(b_{j}\right)$ is (s.s.). Then $\left(b_{j}\right)$ is $(\mathrm{s})$, so $\left(e_{j}\right)$ is $(\mathrm{c})$ by Proposition 2.1 , so in particular $\left(e_{j}\right)$ is a semi-normalized basic sequence. Thus $\left(e_{j}^{*}\right)$ is uniformly bounded. Now let $\left(c_{j}\right)$ be a sequence of scalars with

$$
\mu \stackrel{\mathrm{df}}{=} \sup _{n}\left\|\sum_{j=1}^{n} c_{j} e_{j}\right\|<\infty \text {. }
$$

It then follows that

$$
\sup _{n}\left|c_{n}\right| \leq \sup _{n}\left\|e_{n}^{*}\right\| \mu<\infty
$$

Now define $\left(\alpha_{j}\right)$ by

$$
\alpha_{j}=c_{j}-c_{j+1} \text { for all } j
$$

Then by (6),

$$
\sum_{i=1}^{n} \alpha_{i} b_{i}=\sum_{j=1}^{n+1} c_{j} e_{j}-c_{n+1} b_{n+1} \text { for all } n .
$$

Hence by (15) and (16), $\sup _{n}\left\|\sum_{i=1}^{n} \alpha_{i} b_{i}\right\|<\infty$, whence $\sum \alpha_{i}$ converges, and thus $\left(c_{j}\right)$ converges by (17). Thus $\left(e_{j}\right)$ is (c.c.).

Suppose conversely that $\left(e_{j}\right)$ is (c.c.). Again, $\left(b_{j}\right)$ is $(\mathbf{s})$, so in particular

$\left(b_{j}\right)$ is a semi-normalized basic sequence dominating the summing basis.

Now let $\left(\alpha_{j}\right)$ be a given sequence of scalars with

$$
\sup _{n}\left\|\sum_{j=1}^{n} \alpha_{j} b_{j}\right\|<\infty \text {. }
$$

Let $c_{1}=0$ and $c_{j}=-\sum_{i=1}^{j-1} \alpha_{i}$ for all $j>1$. It then follows by (19) and (20) that

$$
\sup _{n}\left|c_{n}\right|\left\|b_{n}\right\|<\infty .
$$

Now of course $c_{j}-c_{j+1}=\alpha_{j}$ for all $j$; thus by (6),

$$
\sum_{j=1}^{n} c_{j} e_{j}=\sum_{i=1}^{n-1} \alpha_{i} b_{i}+c_{n} b_{n} \text { for all } n .
$$

Hence by (20) and (21), $\sup _{n}\left\|\sum_{j=1}^{n} c_{j} e_{j}\right\|<\infty$, so since $\left(e_{j}\right)$ is (c.c.), $\left(c_{j}\right)$ converges, and thus $\sum \alpha_{i}$ converges. Thus $\left(b_{j}\right)$ is (s.s.).

Our next result shows that with respect to biorthogonal functionals, (s.s.)and (c.c.)-sequences are in perfect duality. 
Proposition 2.4. Let $\left(x_{j}\right)$ be a basic sequence in a Banach space. Then $\left(x_{j}\right)$ is (s.s.) if and only if $\left(x_{j}^{*}\right)$ is (c.c.); $\left(x_{j}\right)$ is (c.c.) if and only if $\left(x_{j}^{*}\right)$ is (s.s.).

Remark. Thus if $\left(x_{j}\right)$ is (s.s.), then in particular $\left(x_{j}^{*}\right)$ is (c), whence by Proposition 2.1, $\left(\sum_{j=1}^{n} x_{j}^{*}\right)_{n=1}^{\infty}$ is an (s)-sequence, and thus a non-trivial weak-Cauchy sequence. This yields Proposition 1.4.

Now in fact, the second statement of Proposition 2.4 follows immediately from the first. Indeed, define $T:\left[x_{j}\right] \rightarrow\left[x_{j}^{*}\right]^{*}$ in the obvious way; $(T x)(f)=$ $f(x)$ for all $f \in\left[x_{j}^{*}\right]^{*}, x \in\left[x_{j}\right]$. Then of course $T$ is an (into) isomorphism and $\left(T x_{j}\right)$ is simply $\left(x_{j}^{* *}\right)$, i.e., the sequence biorthogonal to $\left(x_{j}^{*}\right)$ in $\left[x_{j}^{*}\right]^{*}$. In fact, this "duality-trick" allows us to prove Proposition 2.4 by demonstrating only one implication, by virtue of the following "standard" idea.

Definition 2.3. Let $\mathbf{x}=\left(x_{j}\right)$ be a basic sequence in a Banach space. Set $B(\mathbf{x})=$ $\left\{\left(c_{j}\right):\left(c_{j}\right)\right.$ are scalars with $\left.\sup _{n}\left\|\sum_{j=1}^{n} c_{j} x_{j}\right\|<\infty\right\}$.

Now of course $B(\mathbf{x})$ is a Banach space under the norm

$$
\left\|\left(c_{j}\right)\right\| \stackrel{\mathrm{df}}{=} \sup _{n}\left\|\sum_{j=1}^{n} c_{j} x_{j}\right\| .
$$

In fact $B(\mathbf{x})$ is canonically isomorphic to $\left[x_{j}^{*}\right]^{*}$. Indeed, we define a map $T: B(\mathbf{x}) \rightarrow\left[x_{j}^{*}\right]^{*}$ as follows: $T\left(\left(c_{j}\right)\right)=\sum c_{j} x_{j}^{* *}$, the series converging $\omega^{*}$, where $\left(x_{j}^{* *}\right)$ is the sequence given above. Of course $T$ is just an "extension" of the canonical map already mentioned; since $\left(x_{j}^{* *}\right)$ is a weak*-basis for $\left[x_{j}^{*}\right]^{*}$, it follows easily that $T$ is a surjective isomorphism.

Proof of Proposition 2.4. Let $\left(b_{j}\right)$ be an (s.s.) basic sequence in a Banach space. We first show that $\left(b_{j}^{*}\right)$ is (c.c.). Suppose $\left(c_{j}\right)$ is a sequence of scalars with $\sup _{n}\left\|\sum_{j=1}^{n} c_{j} b_{j}^{*}\right\|<\infty$. It follows that $\sum c_{j} b_{j}^{*}$ converges $\omega^{*}$ to an $f$ in $\left[b_{j}\right]^{*}$. Since $\left(b_{j}\right)$ is weak-Cauchy, $\lim _{j} f\left(b_{j}\right)=\lim _{j} c_{j}$ exists. Now since $\left(b_{j}^{*}\right)$ is a basic sequence, it only remains to show that $\left(\sum_{j=1}^{n} b_{j}^{*}\right)_{n=1}^{\infty}$ is a weak-Cauchy sequence. Letting $T$ be the map defined above, given $f \in\left[b_{j}^{*}\right]^{*}$, choose $\left(c_{j}\right)$ in $B\left(\left(b_{j}\right)\right)$ with $T\left(\left(c_{j}\right)\right)=f$. Then

$$
f\left(\sum_{j=1}^{n} b_{j}^{*}\right)=\sum_{j=1}^{n} c_{j} \text { for all } n ;
$$

hence since $\left(b_{j}\right)$ is (s.s.), $\lim _{n} f\left(\sum_{j=1}^{n} b_{j}^{*}\right)$ exists.

Now to complete the proof of Proposition 2.4 , by the "duality-trick" we need only show that if $\left(e_{j}\right)$ is a (c.c.)-sequence in a Banach space, then $\left(e_{j}^{*}\right)$ is (s.s.). But letting $\left(b_{j}\right)$ be the sequence whose difference sequence is $\left(e_{j}\right)$ (i.e., $b_{n}=\sum_{j=1}^{n} e_{j}$ for all $n$ ), we have that

$$
b_{j}^{*}=e_{j}^{*}-e_{j+1}^{*} \text { for all } j=1,2, \ldots \text {. }
$$


Thus, if $\left(d_{j}\right)$ denotes the difference sequence of $\left(e_{j}^{*}\right)$, then $b_{j}^{*}=-d_{j+1}$ for all $j$. Of course $\left(d_{j}\right)_{j=1}^{\infty}$ is (c.c.) if $-\left(d_{j}\right)_{j=2}^{\infty}$ is. Thus, $\left(e_{j}\right)($ c.c. $) \Rightarrow\left(b_{j}\right)($ s.s. $) \Rightarrow$ $\left(b_{j}^{*}\right)($ c.c. $) \Rightarrow\left(d_{j}\right)($ c.c. $) \Rightarrow\left(e_{j}^{*}\right)$ (s.s.) .

Remark. We may define a semi-normalized basic sequence $\left(x_{j}\right)$ in a Banach space to be wide-(s.s.) (resp. wide-(c.c.)) if whenever $\left(c_{j}\right)$ is a given sequence of scalars with $\sup _{n}\left\|\sum_{j=1}^{n} c_{j} x_{j}\right\|<\infty$, then $\sum c_{j}$ converges (resp. $\left(c_{j}\right)$ converges and $\left.\sup _{n}\left\|\sum_{i=1}^{n} x_{i}\right\|<\infty\right)$. Then the arguments for Propositions 2.3 and 2.4 yield the following generalization:

Proposition. Let $\left(b_{j}\right)$ be a semi-normalized basic sequence, with difference sequence $\left(e_{j}\right)$.

(a) $\left(b_{j}\right)$ is wide-(s.s.) if and only if $\left(e_{j}\right)$ is wide-(c.c.).

(b) $\left(b_{j}\right)$ is (s) if and only if $\left(b_{j}^{*}\right)$ is wide-(c.c.).

(c) $\left(b_{j}\right)$ is wide-(s.s.) if and only if $\left(b_{j}^{*}\right)$ is (c).

We continue with further permanence properties.

Proposition 2.5. Let $\left(x_{j}\right)$ be an (s.s.)-sequence. Then every convex block basis of $\left(x_{j}\right)$ is also (s.s.).

Proof. Let $\left(y_{j}\right)$ be a convex block basis of $\left(x_{j}\right)$. Choose $0 \leq n_{1}<n_{2}<\cdots$ and scalars $\left(\lambda_{i}\right)$ so that for all $j$,

$$
y_{j}=\sum_{i=n_{j}+1}^{n_{j+1}} \lambda_{i} x_{i} \text { with } \lambda_{i} \geq 0 \text { for all } i \text { and } \sum_{i=n_{j}+1}^{n_{j+1}} \lambda_{i}=1 \text {. }
$$

Now it follows easily that $\left(y_{j}\right)$ is a weak-Cauchy basic sequence, since $\left(x_{j}\right)$ is. Let scalars $\left(c_{j}\right)$ be given with $\sup _{n}\left\|\sum_{j=1}^{n} c_{j} y_{j}\right\| \stackrel{\text { df }}{=} \mu<\infty$, and let $K$ be the basis-constant of $\left(x_{j}\right)$. Define $\left(\alpha_{i}\right)$ by $\alpha_{i}=c_{j} \lambda_{i}$ for $n_{j}<i \leq n_{j+1}$, $j=1,2, \ldots$. Then $\left\|\sum_{r=1}^{i} \alpha_{r} x_{r}\right\| \leq K \mu$ for all $i$. Hence $\sum_{i=1}^{\infty} \alpha_{i}$ converges, to $s$ say. So in particular, $\lim _{j \rightarrow \infty} \sum_{i=1}^{n_{j+1}} \alpha_{i}=s$. But fixing $j$,

$$
\sum_{i=1}^{n_{j+1}} \alpha_{i}=\sum_{k=1}^{j} \sum_{i=n_{k}+1}^{n_{k+1}} c_{k} \lambda_{i}=\sum_{k=1}^{j} c_{k}
$$

Thus $\sum c_{k}$ converges.

Our next result follows from our main theorem and known results. However its direct proof is quite simple, so we give this here.

Proposition 2.6. Let $X$ be a Banach space containing no isomorph of $\ell^{1}$, and suppose $X^{*}$ is not weakly sequentially complete. Then $X$ has an (s.s.)-sequence.

Remark. As noted following Corollary 1.2, in fact (using Theorem 1.1), we also have that every non-trivial weak-Cauchy sequence in $X^{*}$ has an (s.s.)subsequence. 
Proof of 2.6. We first note the following simple fact: Let $X, Y$ be Banach spaces, $T: X \rightarrow Y$ a bounded linear operator, and $\left(x_{j}\right)$ a (wcb) sequence in $X$ such that $\left(T x_{j}\right)$ is (s.s.). Then $\left(x_{j}\right)$ is (s.s.).

Now let $\left(f_{n}\right)$ be a non-trivial weak-Cauchy sequence in $X^{*}$. In particular, there is an $f$ in $X^{*}$ so that $f_{n} \rightarrow f \omega^{*}$. But then $\left(f_{n}-f\right)$ is also non-trivial weak-Cauchy, and hence has an (s)-subsequence by Proposition 2.1. That is, we have

$$
\text { there is an (s)-sequence }\left(f_{n}\right) \text { in } X^{*} \text { with }\left(f_{n}\right) \omega^{*} \text {-null. }
$$

Next, we may assume without loss of generality that $X$ is separable. We then deduce, by a result in [JR] (cf. also [R3]), that $\left(f_{n}\right)$ has a weak*-basic subsequence, so without loss of generality, let us assume that $\left(f_{n}\right)$ itself is weak*-basic. It follows that there is a Banach space $Y$ with a basis $\left(y_{j}\right)$ and a bounded linear surjection $T: X \rightarrow Y$ so that

$$
T^{*} y_{j}^{*}=f_{j} \text { for all } j \text {. }
$$

Then letting $\left(f_{j}^{*}\right)$ be the functionals biorthogonal to $\left(f_{j}\right)$ (in $\left.\left[f_{j}\right]^{*}\right)$, it follows from (25) (since $T^{*}$ is an into-isomorphism) that

$$
\left(y_{j}\right) \text { is equivalent to }\left(f_{j}^{*}\right) \text {. }
$$

Now by the remark following the proof of Proposition 2.4, since $\left(f_{j}\right)$ is an (s)-sequence, $\left(f_{j}^{*}\right)$ is wide-(c.c.). (In fact the first part of the proof of 2.4 yields this immediately.) Thus by $(26),\left(y_{j}\right)$ is wide- (c.c.), so by the proof of Proposition 2.3, setting $u_{n}=\sum_{j=1}^{n} y_{j}$ for all $n$, we have that $\left(u_{n}\right)$ is wide(s.s.). Now since $\ell^{1}$ doesn't embed in $X$, it doesn't embed in $Y$ either. Hence $\left(u_{n}\right)$ has a weak-Cauchy subsequence $\left(u_{n}^{\prime}\right)$. But then $\left(u_{n}^{\prime}\right)$ is an (s.s.)sequence. Now by the open mapping theorem, we may choose $\left(b_{j}\right)$ a bounded sequence in $X$ with $T b_{j}=u_{j}^{\prime}$ for all $j$. Now of course $\left(b_{j}\right)$ has no weakly convergent subsequence, since $\left(u_{j}^{\prime}\right)$ is itself non-trivial weak-Cauchy. Thus by the $\ell^{1}$-Theorem and Proposition 2.2, we may choose $\left(x_{j}\right)$ an (s)-sequence with $\left(x_{j}\right)$ a subsequence of $\left(b_{j}\right)$. Since $\left(T x_{j}\right)$ is a subsequence of $\left(u_{j}^{\prime}\right),\left(T x_{j}\right)$ is (s.s.), so by the fact mentioned at the beginning of the proof, $\left(x_{j}\right)$ is (s.s.).

Remark. Proposition 2.6 yields another proof of the known result: if $\ell^{1}$ does not embed in $X$ and $X$ has property $(u)$, then $X^{*}$ is weakly sequentially complete. (This result follows immediately from the $\ell^{1}$-Theorem and results of A. Petczyński [P2].) Indeed, if not, then since $X$ has an (s.s.)-sequence, our argument for Corollary 1.5 shows that $X$ fails property $(u)$, a contradiction.

The final two results of this section will be used as tools in the proof of the main theorem. The first one gives an equivalence for (c.c.)-sequences; its proof follows by an argument of $\mathrm{S}$. Bellenot [Be].

Proposition 2.7. Let $\left(e_{j}\right)$ be a given (c)-sequence in a Banach space. Then the following are equivalent.

(a) $\left(e_{j}\right)$ is a (c.c.)-sequence. 
(b) For any sequence of scalars $\left(c_{j}\right)$ with $c_{j}=0$ for infinitely many $j$ and $\sup _{n}\left\|\sum_{j=1}^{n} c_{j} e_{j}\right\|<\infty, c_{j} \rightarrow 0$ as $j \rightarrow \infty$.

Remarks. 1. Call $\left(x_{j}\right)$ a proper subsequence of $\left(e_{j}\right)$ if the $x_{j}$ 's are not ultimately the $e_{j}$ 's; that is, there exist $n_{1}<n_{2}<\cdots$ with $\mathbb{N} \sim\left\{n_{1}, n_{2}, \ldots\right\}$ infinite, with $x_{j}=e_{n_{j}}$ for all $j$. Condition (b) may then be reformulated: Every proper subsequence of $\left(e_{j}\right)$ is semi-boundedly complete.

2: The proof of 2.7 yields also that if $\left(e_{j}\right)$ is a given wide- (c) sequence, then $\left(e_{j}\right)$ is wide-(c.c.) if and only if (b) holds.

Proof of 2.7. (a) $\Rightarrow$ (b) is trivial; we show (b) $\Rightarrow$ (a). Let $\left(c_{j}\right)$ be a sequence of scalars with $\sup _{n}\left\|\sum_{j=1}^{n} c_{j} e_{j}\right\|<\infty$. We must show that $\left(c_{j}\right)$ converges. Since $\left(e_{j}\right)$ is a semi-normalized basic sequence, $\left(c_{j}\right)$ is a bounded sequence, and hence has a convergent subsequence. Thus we may choose $n_{1}<n_{2}<\cdots$ and $c$ so that

$$
\left|c_{n_{j}}-c\right|<\frac{1}{2^{j}} \text { for all } j .
$$

Now define sequences $\left(\alpha_{i}\right)$ and $\left(\beta_{i}\right)$ by $\alpha_{i}=c_{i}-c$ if $i \neq n_{j}$ for any $j ; \alpha_{n_{j}}=0$ for $j=1,2, \ldots ; \beta_{i}=0$ if $i \neq n_{j}$ for any $j ; \beta_{n_{j}}=c_{n_{j}}-c$ for $j=1,2, \ldots$. We have that since $\sup _{n}\left\|\sum_{i=1}^{n} e_{i}\right\|<\infty, \sup _{n}\left\|\sum_{i=1}^{n}\left(c_{i}-c\right) e_{i}\right\|<\infty$, and hence since

and

$$
\sum\left\|\beta_{i} e_{i}\right\|<\sum \frac{1}{2^{j}}\left\|e_{n_{j}}\right\|<\infty \text { by }(27)
$$

$$
\begin{gathered}
\sum_{i=1}^{n} \alpha_{i} e_{i}=\sum_{i=1}^{n}\left(c_{i}-c\right) e_{i}-\sum_{i=1}^{n} \beta_{i} e_{i} \text { for all } n, \\
\sup _{n}\left\|\sum_{i=1}^{n} \alpha_{i} e_{i}\right\|<\infty .
\end{gathered}
$$

Since $\alpha_{i}=0$ for infinitely many $i,(28)$ yields that $\lim _{i \rightarrow \infty} \alpha_{i}=0$. But in virtue of (27), this implies that $\lim _{i \rightarrow \infty} c_{i}=c$.

Our last result of this section gives a criterion for extracting (s.s.)-subsequences from a given sequence, which we use directly in the proof of Theorem 1.1. It is convenient to first give the following $\varepsilon$-version of (c.c.)-sequences.

Definition 2.4. A (c)-sequence $\left(e_{j}\right)$ in a Banach space is called an $\varepsilon$-(c.c.)sequence if whenever $\left(c_{j}\right)$ is a sequence of scalars with $c_{j}=0$ for infinitely many $j$ and $\left\|\sum_{j=1}^{n} c_{j} e_{j}\right\| \leq 1$ for all $n$, then $\varlimsup_{j \rightarrow \infty}\left|c_{j}\right| \leq \varepsilon$.

Lemma 2.8. Let $\left(f_{j}\right)$ be an (s)-sequence in a Banach space. Then $\left(f_{j}\right)$ has an (s.s.)-subsequence provided, for every $\varepsilon>0$ and subsequence $\left(g_{j}\right)$ of $\left(f_{j}\right)$, there is a subsequence $\left(b_{j}\right)$ of $\left(g_{j}\right)$ whose difference sequence $\left(e_{j}\right)$ is an $\varepsilon$ - (c.c.)sequence.

Lemma 2.8 follows immediately, by diagonalization, from Proposition 2.7 and the following quantitative permanence properties. 
P1. Let $\left(b_{j}\right)$ be an (s)-sequence. There exists $a \lambda<\infty$ so that if $\left(b_{j}^{\prime}\right)$ is a subsequence of $\left(b_{j}\right)$, then the difference sequence $\left(e_{j}^{\prime}\right)$ of $\left(b_{j}^{\prime}\right)$ is $\lambda$-basic.

Indeed, the proof of Proposition 2.1 yields that if $\left(b_{j}\right)$ has basis-constant $\beta$, and $\mathbf{s}$ is its summing functional, then $\left(e_{j}^{\prime}\right)$ as above is $\lambda$-basic where $\lambda=$ $\beta+(1+\beta)\|\mathbf{s}\| \sup _{k}\left\|b_{k}\right\|$.

P2. Let $\left(b_{j}\right)$ be an (s)-sequence, $\lambda$ as in $\mathrm{P} 1$, and $\varepsilon>0$. Then if the difference sequence $\left(e_{j}\right)$ of $\left(b_{j}\right)$ is $\varepsilon$-(c.c.) and $\left(b_{j}^{\prime}\right)$ is a subsequence of $\left(b_{j}\right)$ with difference sequence $\left(e_{j}^{\prime}\right)$, then $\left(e_{j}^{\prime}\right)$ is $\lambda \varepsilon$ - (c.c.).

To see this, suppose $\left(c_{j}\right)$ is a sequence of scalars with $\left\|\sum_{j=1}^{k} c_{j} e_{j}^{\prime}\right\| \leq 1$ for all $k$ and $c_{j}=0$ for infinitely many $j$. Suppose $n_{1}<n_{2}<\cdots$ are chosen with $b_{j}^{\prime}=b_{n_{j}}$ for all $j$. Then setting $n_{0}=0$,

$$
e_{j}^{\prime}=\sum_{i=n_{j-1}+1}^{n_{j}} e_{i} \text { for all } j
$$

Now define $\left(\alpha_{i}\right)$ by $\alpha_{i}=c_{j}$ if $n_{j-1}<i \leq n_{j}$, for all $j$. Then it follows that

$$
\left\|\sum_{i=1}^{k} \alpha_{i} e_{i}\right\| \leq \lambda \text { for all } k
$$

and of course $\alpha_{i}=0$ for infinitely many $i$, whence $\varlimsup_{i}\left|\alpha_{i}\right|=\varlimsup_{j}\left|c_{j}\right| \leq$ $\lambda \varepsilon$.

\section{DIFFERENCES OF BOUNDED SEMI-CONTINUOUS FUNCTIONS}

We first prove the (known) fundamental connection between sequences equivalent to the summing basis and differences of bounded semi-continuous functions, Proposition 1.7. We need some basic permanence properties of DUCsequences (cf. Definition 1.2).

Suppose $\left(x_{j}\right)$ is a WUC-sequence in a Banach space $X$. A simple application of the uniform boundedness principle yields that there is a $K<\infty$ so that $\sum_{j=1}^{\infty}\left|x^{*}\left(x_{j}\right)\right| \leq K\left\|x^{*}\right\|$ for all $x^{*} \in X^{*}$. We accordingly define $\left\|\left(x_{j}\right)\right\|_{\mathrm{wuc}}$ by

$$
\left\|\left(x_{j}\right)\right\|_{\mathrm{WUC}}=\sup \left\{\sum_{j=1}^{\infty}\left|x^{*}\left(x_{j}\right)\right|: x^{*} \in B a\left(X^{*}\right)\right\} .
$$

Similarly, if $\left(y_{j}\right)$ is a DUC-sequence in $X$, we define $\left\|\left(y_{j}\right)\right\|_{\text {DUC }}$ by

$$
\left\|\left(y_{j}\right)\right\|_{\mathrm{DUC}}=\left\|\left(y_{j}-y_{j-1}\right)_{j=1}^{\infty}\right\|_{\mathrm{WUC}}
$$

(where we set $y_{0}=0$ ).

The next result now readily follows from the basic structure of (s)-sequences. 
Proposition 3.1. A sequence in a Banach space is equivalent to the summing basis if and only if it is a DUC-(s)-sequence.

Proof. It is trivial that the summing basis is both DUC and (s); hence so is any sequence equivalent to it. Suppose conversely that $\left(b_{j}\right)$ is a DUC-(s)-sequence, with difference sequence $\left(e_{j}\right)$. Thus $\left(e_{j}\right)$ is WUC and (by Proposition 2.1) a basic sequence. Let then $K=\left\|\left(e_{j}\right)\right\|_{\mathrm{Wuc}}$ and $\lambda=\sup _{j}\left\|e_{j}^{*}\right\|$ where $\left(e_{j}^{*}\right)$ are the biorthogonal functionals to $\left(e_{j}\right)$. Then given $n$, scalars $c_{1}, \ldots, c_{n}$, and $x^{*} \in B a\left(X^{*}\right)$, we have that

$$
\left|x^{*}\left(\sum_{i=1}^{n} c_{i} e_{i}\right)\right| \leq \max _{i}\left|c_{i}\right| \sum_{i=1}^{n}\left|x^{*}\left(e_{i}\right)\right| \leq K \max _{i}\left|c_{i}\right| .
$$

Hence

$$
\frac{1}{\lambda} \max _{i}\left|c_{i}\right| \leq\left\|\sum_{i=1}^{n} c_{i} e_{i}\right\| \leq K \max _{i}\left|c_{i}\right| .
$$

Of course (33) yields that $\left(e_{i}\right)$ is equivalent to the $c_{0}$-basis, whence $\left(b_{i}\right)$ is equivalent to the summing basis.

We next give some permanence properties of DUC-sequences.

Proposition 3.2. Let $\left(x_{j}\right),\left(y_{j}\right)$ be given sequences in a Banach space.

(a) If $\left(x_{j}\right),\left(y_{j}\right)$ are DUC, so are $\left(x_{j}+y_{j}\right)$ and $\left(\lambda x_{j}\right)$ for any scalar $\lambda$.

(b) If $\left(x_{j}\right)$ is DUC and $\left(y_{j}\right)$ is a convex block basis of $\left(x_{j}\right)$, then $\left(y_{j}\right)$ is DUC and moreover

$$
\left\|\left(y_{j}\right)\right\|_{\mathrm{DUC}} \leq\left\|\left(x_{j}\right)\right\|_{\mathrm{DUC}} .
$$

Remark. (a) is trivial, (b) is not. Of course the immediate argument for (a) yields that the DUC-sequences form a normed linear space under $\|\cdot\|_{D U C}$, and, with a little more work, a Banach space. (In fact, of course the space of DUCsequences is isometric to that of the WUC sequences; but the latter is in turn isometric to $\mathscr{L}\left(c_{0}, X\right)$.) Note that if $\left(x_{j}\right)$ is DUC and $\sum\left\|x_{j}-y_{j}\right\|<\infty$, then since $\left(x_{j}-y_{j}\right)$ is DUC, so is $\left(y_{j}\right)$. We shall apply (a) in this form.

Proof of 3.2 (b). Let $e_{1}=x_{1}, e_{j}=x_{j}-x_{j-1}$ for $j>1$. Then it follows that given $k<\ell$ and scalars $\lambda_{k+1}, \ldots, \lambda_{\ell}$ with $\sum_{j=k+1}^{\ell} \lambda_{j}=1$ and $y=$ $\sum_{i=k+1}^{\ell} \lambda_{i} x_{i}$, then setting $\rho_{j}=\sum_{i=j}^{\ell} \lambda_{i}$ for all $k<j \leq \ell$, we have that $\rho_{k+1}=1$ and

$$
y=\sum_{i=1}^{k} e_{i}+\sum_{j=k+1}^{\ell} \rho_{j} e_{j} .
$$

Hence also given $m>\ell$ and scalars $\lambda_{\ell+1}, \ldots, \lambda_{m}$ with $\sum_{j=\ell+1}^{m} \lambda_{j}=1$ and $\bar{y}=\sum_{i=\ell+1}^{m} \lambda_{i} x_{i}$, then setting $\bar{\rho}_{j}=\sum_{i=j}^{m} \lambda_{i}$ for all $\ell<j \leq m$, we have that

$$
\bar{y}-y=\sum_{j=k+1}^{\ell}\left(1-\rho_{j}\right) e_{j}+\sum_{j=\ell+1}^{m} \bar{\rho}_{j} e_{j} \text {. }
$$


Now let $\left(y_{i}\right)$ be a convex block basis of $\left(x_{i}\right)$. Then we may choose $0=n_{0}<$ $n_{1}<n_{2}<\cdots$ and non-negative scalars $\lambda_{1}, \lambda_{2}, \ldots$ so that, for all $i$,

$$
y_{i}=\sum_{j=n_{i-1}+1}^{n_{i}} \lambda_{j} x_{j} \text { and } \sum_{j=n_{i-1}+1}^{n_{i}} \lambda_{j}=1 .
$$

Then setting $\rho_{j}^{i}=\sum_{k=j}^{n_{i}} \lambda_{k}$ for all $n_{i-1}<j \leq n_{i}$, it follows by (35) that

$$
\begin{aligned}
y_{1} & =\sum_{j=1}^{n_{1}} \rho_{j}^{1} e_{j} \text { and } \\
y_{i+1}-y_{i} & =\sum_{j=n_{i-1}+1}^{n_{i}}\left(1-\rho_{j}^{i}\right) e_{j}+\sum_{j=n_{i}+1}^{n_{i+1}} \rho_{j}^{i} e_{j} \text { for all } i .
\end{aligned}
$$

Now setting $y_{0}=0$ for convenience and letting $x^{*} \in B a X^{*}$, we have by (36) and the fact that $0 \leq \rho_{j}^{i} \leq 1$ for all $i$ and $j$ that

$$
\begin{aligned}
& \sum_{i=0}^{\infty}\left|x^{*}\left(y_{i+1}\right)-x^{*}\left(y_{i}\right)\right| \\
& \quad \leq \sum_{i=1}^{\infty} \sum_{j=n_{i-1}+1}^{n_{i}} \rho_{j}^{i}\left|x^{*}\left(e_{j}\right)\right|+\sum_{i=1}^{\infty} \sum_{j=n_{i-1}+1}^{n_{i}}\left(1-\rho_{j}^{i}\right)\left|x^{*}\left(e_{j}\right)\right| \\
& \quad=\sum_{j=1}^{\infty}\left|x^{*}\left(e_{j}\right)\right| \leq\left\|\left(e_{j}\right)\right\|_{\mathrm{WUC}}=\left\|\left(x_{j}\right)\right\|_{\mathrm{DUC}} \cdot
\end{aligned}
$$

Corollary 3.3. A non-weakly convergent DUC-sequence has a subsequence equivalent to the summing basis.

Proof. Let $\left(x_{j}\right)$ be such a sequence. Then (since $\left(x_{j}-x_{j-1}\right)$ is WUC) it follows that $\left(x_{j}\right)$ is non-trivial weak-Cauchy. Thus by Proposition 2.2, $\left(x_{j}\right)$ has an (s)subsequence $\left(y_{j}\right)$. Of course $\left(y_{j}\right)$ is then a convex block basis of $\left(x_{j}\right)$; hence $\left(y_{j}\right)$ is also DUC by the previous result, so $\left(y_{j}\right)$ is equivalent to the summing basis by Proposition 3.1.

We are now prepared for the

Proof of Proposition 1.7. Let $f,\left(f_{n}\right)$, and $K$ be as in the statement of the proposition. Of course we work in the Banach space $X=C(K)$. Suppose first that $\left(g_{n}\right)$ is a convex block basis of $\left(f_{n}\right)$, with $\left(g_{n}\right)$ equivalent to the summing basis. But then it follows that setting $g_{0}=0, g_{n} \rightarrow f$ pointwise, and for all $k \in K$,

$$
\sum_{n=1}^{\infty}\left|g_{n}(k)-g_{n-1}(k)\right| \leq\left\|\left(g_{n}\right)\right\|_{\mathrm{DUC}}<\infty \text {. }
$$


Hence $f$ is in $D(K)$. Now suppose conversely that $f$ is in $D(K)$. We may then choose $C<\infty$ and $\varphi_{1}, \varphi_{2}, \ldots$ in $C(K)$ with

$$
\sum_{j=1}^{\infty}\left|\varphi_{j}(k)\right| \leq C \text { and } \sum_{j=1}^{\infty} \varphi_{j}(k)=f(k) \text { for all } k \in K .
$$

Now set $g_{j}=\sum_{i=1}^{j} \varphi_{i}$ for all $j$. Then it follows that $f_{j}-g_{j} \rightarrow 0$ pointwise as $j \rightarrow \infty$, and hence $f_{j}-g_{j} \rightarrow 0$ weakly in $C(K)$, since $\left(f_{j}\right),\left(g_{j}\right)$ are bounded sequences. Thus we may choose $0 \leq n_{1}<n_{2}<\cdots$ positive integers and non-negative scalars $\lambda_{1}, \lambda_{2}, \ldots$ with

$$
\sum_{i=n_{j}+1}^{n_{j+1}} \lambda_{i}=1 \text { and }\left\|\sum_{i=n_{j}+1}^{n_{j+1}} \lambda_{i}\left(f_{i}-g_{i}\right)\right\| \leq \frac{1}{2^{j}} \text { for all } j .
$$

Now set $u_{j}=\sum_{i=n_{j}+1}^{n_{j+1}} \lambda_{i} f_{i}$ and $v_{j}=\sum_{i=n_{j}+1}^{n_{j+1}} \lambda_{i} g_{i}$ for all $j$. Of course it follows from (38) that $\left(v_{j}\right)$ is a convex block basis of $\left(g_{i}\right)$, and since (37) immediately yields that $\left\|\left(g_{i}\right)\right\|_{\mathrm{DUC}} \leq C<\infty$, Proposition $3.2 \mathrm{~b}$ yields that $\left(v_{i}\right)$ is DUC. Hence also by $3.2 \mathrm{a}$ and (38), $\left(u_{j}\right)$ is DUC (in fact $\left.\left\|\left(u_{j}\right)\right\|_{\mathrm{DUC}} \leq C+1\right)$, and again by (38), $u_{j} \rightarrow f$ pointwise. Since $f$ is discontinuous, $\left(u_{j}\right)$ is nonweakly convergent and hence has a subsequence $\left(u_{j}^{\prime}\right)$ equivalent to the summing basis by Corollary 3.3. This completes the proof, since $\left(u_{j}^{\prime}\right)$ is again a convex block basis of $\left(f_{i}\right)$.

We now treat some basic intrinsic invariants of differences of bounded semicontinuous functions. It is convenient to define these on an arbitrary separable metric space $K$. We let $C(K)$ denote the space of continuous complex-valued functions on $X$ and $C_{b}(K)$ the space of bounded members of $C(K)$ under the sup norm. Now we define $D(K)$ exactly as in Definition 1.5 in the compact case. It is not hard to show that $D(K)$ is then a Banach space, where we define $\|\cdot\|_{D}$ on $D(K)$ by

$$
\begin{aligned}
\|f\|_{D}=\inf \left\{\sup _{k \in K} \sum_{j=1}^{\infty}\left|\varphi_{j}(k)\right|: \varphi_{j} \in C_{b}(K) \text { all } j\right. \\
\text { and } \left.f=\sum \varphi_{j} \text {-pointwise }\right\} .
\end{aligned}
$$

Now if $f \geq 0$ is bounded and lower semi-continuous, a result of Baire's gives that there exist continuous $f_{j}$ 's with $0 \equiv f_{0} \leq f_{1} \leq f_{2} \leq f_{3} \cdots$ and $f_{n} \rightarrow f$ pointwise. Of course then $f=\sum_{j=1}^{\infty}\left(f_{j}-f_{j-1}\right)$ so we obtain that $\|f\|_{D}=\|f\|_{\infty}$. If $f$ is real-valued and in $D(K)$, then it follows that

$$
\begin{aligned}
& \|f\|_{D}=\inf \left\{\|u+v\|_{\infty}: f=u-v, u, v \geq 0\right. \text { are bounded } \\
& \text { lower semi-continuous\}. }
\end{aligned}
$$

We prove below the rather surprising result that this infimum is attained.

We introduce a new concept here, that of the transfinite oscillations of a given function. These will be our basic tool in studying $D(K)$. They are closely 
related to earlier transfinite invariants defined by A.S. Kechris and A. Louveau [KL], which we term here the positive transfinite oscillations (see Definition 3.2 and the following below). We first recall the upper and lower semi-continuous envelopes of a given extended real-valued function $f$ on $X: U f$, the upper semi-continuous envelope of $f$, is defined by

$$
U f(x)=\varlimsup_{y \rightarrow x} f(y) \text { for all } x \in X ;
$$

similarly, $L f$, the lower semi-continuous envelope of $f$, is defined by

$$
(L f)(x)=\varliminf_{y \rightarrow x} f(y) \text { for all } x \in K .
$$

Now if $f: X \rightarrow \mathbb{C}$ is a given function, we define osc $f$, the oscillation of $f$, as follows: First, we set

$$
\underline{\operatorname{osc}} f(x)=\varlimsup_{y \rightarrow x}|f(y)-f(x)| \text { for all } x \in K .
$$

Then we set

$$
\operatorname{osc} f=U \underline{\text { osc }} f .
$$

Now it is easily seen that if $f$ is real-valued, then

$$
\text { osc } f=\max \{U f-f, f-L f\} \text {. }
$$

osc $f$ is not in general upper semi-continuous; nevertheless this invariant is more appropriate for our purposes than the usual definition of the oscillation, which we term $\overline{\operatorname{osc}} f$, the upper oscillation of $f$ :

$$
\overline{\operatorname{osc}} f(x)=\varlimsup_{y, z \rightarrow x}|f(y)-f(z)|
$$

( $=U f-L f$ if $f$ is extended real-valued). It's worth pointing out that if $f$ is bounded complex-valued, then $\|\operatorname{osc} f\|_{\infty} \leq\|\overline{\text { osc }} f\|_{\infty} \leq 2\|f\|_{\infty}$ while if $f$ is non-negative, then $\|\overline{\text { osc }} f\|_{\infty}=\|U f-L f\|_{\infty} \leq\|U f\|_{\infty}=\|f\|_{\infty}$.

Definition 3.1. Let $f: K \rightarrow \mathbb{C}$ be a given function, $K$, a separable metric space, $\alpha$ a countable ordinal. We define the $\alpha^{\text {th }}$ oscillation of $f, \operatorname{osc}_{\alpha} f$, by induction, as follows: set $\operatorname{osc}_{0} f \equiv 0$. Suppose $\beta>0$ is a countable ordinal, and $\operatorname{osc}_{\alpha} f$ has been defined for all $\alpha<\beta$. If $\beta$ is a successor, say $\beta=\alpha+1$, we define

$$
\widetilde{\operatorname{osc}}_{\beta} f(x)=\varlimsup_{y \rightarrow x}\left(|f(y)-f(x)|+\operatorname{osc}_{\alpha} f(y)\right) \text { for all } x \in K .
$$

If $\beta$ is a limit ordinal, we set

$$
\widetilde{\mathrm{osc}}_{\beta} f=\sup _{\alpha<\beta} \operatorname{osc}_{\alpha} f .
$$

Finally, we set $\operatorname{osc}_{\beta} f=U \widetilde{\operatorname{osc}_{\beta}} f$.

Evidently we have that $\widetilde{\text { osc }} 1=\underline{\operatorname{osc}} f$ and $\operatorname{osc}_{1} f=\operatorname{osc} f$. Next we list some useful permanence properties of the transfinite oscillations of a function. 
Proposition 3.4. Let $f, g$ be given complex-valued functions on $K$, a separable metric space, $t$ a complex number, and $\alpha, \beta$ non-zero countable ordinals.

(a) osc $_{\alpha} f$ is an upper semi-continuous $[0, \infty]$-valued function; if $\alpha \leq \beta$, then $\operatorname{osc}_{\alpha} f \leq \operatorname{osc}_{\beta} f$.

(b) $\operatorname{osc}_{\alpha} t f=|t| \operatorname{osc}_{\alpha} f$ and $\operatorname{osc}_{\alpha}(f+g) \leq \operatorname{osc}_{\alpha} f+\operatorname{osc}_{\alpha} g$.

(c) If $\operatorname{osc}_{\alpha} f=\operatorname{osc}_{\alpha+1} f$, then $\operatorname{osc}_{\alpha} f=\operatorname{osc}_{\beta} f$ for all $\beta>\alpha$. Moreover if $f$ is real valued, this happens if and only if $\operatorname{osc}_{\alpha} f \pm f$ are both upper semi-continuous functions.

(d) If $f$ is semi-continuous, then $\operatorname{osc}_{\alpha} f=\operatorname{osc} f$.

Proof. The assertions up to the "moreover" statement in (c) are easily proved by transfinite induction, using Definition 3.1.

To prove this part of (c), we first note that $\operatorname{osc}_{\alpha} f \leq \widetilde{\mathrm{osc}}_{\alpha+1} f \leq \operatorname{osc}_{\alpha+1} f$. It then follows that

$$
\operatorname{osc}_{\alpha+1} f=\operatorname{osc}_{\alpha} f \text { if and only if } \widetilde{\text { osc }}_{\alpha+1} f=\operatorname{osc}_{\alpha} f
$$

(for if the latter equality holds, then since osc $f$ is upper semi-continuous, osc $_{\alpha+1} f=U \widetilde{\mathrm{osc}}_{\alpha+1} f=U \mathrm{osc}_{\alpha} f=\mathrm{osc}_{\alpha} f$ ).

Now assume $f$ is real valued, and suppose first that osc $f=$ osc $_{\alpha+1} f$. To see that $f+\operatorname{osc}_{\alpha} f$ is upper semi-continuous, let $x \in K$ and $\left(y_{n}\right)$ a sequence in $K$ with $y_{n} \rightarrow x$. Then

$$
\begin{aligned}
\varlimsup_{n \rightarrow \infty} & f\left(y_{n}\right)-f(x)+\operatorname{osc}_{\alpha} f\left(y_{n}\right) \\
& \leq \varlimsup_{n \rightarrow \infty}\left|f\left(y_{n}\right)-f(x)\right|+\operatorname{osc}_{\alpha} f\left(y_{n}\right) \\
& \leq \widetilde{\text { osc }}_{\alpha+1} f(x)=\operatorname{osc}_{\alpha} f(x) \text { by (48). }
\end{aligned}
$$

Hence

$$
\varlimsup_{n \rightarrow \infty} f\left(y_{n}\right)+\operatorname{osc}_{\alpha} f\left(y_{n}\right) \leq f(x)+\operatorname{osc}_{\alpha} f(x),
$$

proving $f+\operatorname{osc}_{\alpha} f$ is upper semi-continuous. Since osc $\beta=\operatorname{osc}_{\beta}-f$ for all $\beta$ by 3.4(b), it follows immediately upon replacing $f$ by $-f$ that also $f-\operatorname{osc}_{\alpha} f$ is upper semi-continuous.

Now suppose conversely that $\operatorname{osc}_{\alpha} f \pm f$ are upper semi-continuous, yet osc $_{\alpha+1} f \neq \operatorname{osc}_{\alpha} f$. Then by (48) we may choose $x \in K$ so that $\widetilde{\mathrm{osc}}_{\alpha+1} f(x)>$ $\operatorname{osc}_{\alpha} f(x)$. But

$$
\begin{aligned}
& \widetilde{0 s c}_{\alpha+1} f(x)=\varlimsup_{y \rightarrow x}|f(y)-f(x)|+\operatorname{osc}_{\alpha} f(y) \\
& \quad=\max \left\{\varlimsup_{y \rightarrow x}(f(y)-f(x))+\operatorname{osc}_{\alpha} f(y), \varlimsup_{y \rightarrow x}(f(x)-f(y))+\text { osc }_{\alpha} f(y)\right\} .
\end{aligned}
$$

Thus either

$$
\varlimsup_{y \rightarrow x} f(y)-f(x)+\operatorname{osc}_{\alpha} f(y)>\text { osc }_{\alpha} f(x)
$$

or

$$
\varlimsup_{y \rightarrow x} f(x)-f(y)+\operatorname{osc}_{\alpha} f(y)>\operatorname{osc}_{\alpha} f(x) .
$$


But if (49i) holds, $f+$ osc $_{\alpha} f$ is not upper semi-continuous, while if (49ii) holds, $(-f)+$ osc $_{\alpha} f$ is not upper semi-continuous.

Finally, to prove $3.4(\mathrm{~d})$, suppose without loss of generality that $f$ is upper semi-continuous. (For if $f$ is lower semi-continuous, $-f$ is upper semicontinuous, and $\operatorname{osc}_{\alpha} f=\operatorname{osc}_{\alpha}-f$.) But then $f=U f$ and hence

$$
\text { osc } f=f-L f=\operatorname{osc} f \quad(=\overline{\operatorname{osc}} f)
$$

(since $-L f$ is upper semi-continuous). But then osc $f+f=f-L f+f=$ $2 f-L f$ and osc $f-f=-L f$; thus osc $f \pm f$ are both upper semi-continuous, so (d) follows from (c).

We may now formulate our main structural result concerning $D(K)$.

Theorem 3.5. Let $K$ be a separable metric space and $f: K \rightarrow \mathbb{C}$ a bounded function. There exists a countable ordinal $\alpha$ so that $\operatorname{osc}_{\alpha} f=\operatorname{osc}_{\beta} f$ for all $\beta>\alpha$. Letting $\tau$ be the least such $\alpha$, then $f$ is in $D(K)$ if and only if $\operatorname{osc}_{\tau} f$ is bounded. When $f$ is real valued and this occurs, then

$$
\|f\|_{D}=\left\||f|+\operatorname{osc}_{\tau} f\right\|_{\infty} \text {. }
$$

Moreover setting $\lambda=\left\||f|+\operatorname{osc}_{\tau} f\right\|_{\infty}$,

$$
u=\frac{\lambda-\mathrm{osc}_{\tau} f+f}{2} \text { and } v=\frac{\lambda-\mathrm{osc}_{\tau} f-f}{2},
$$

$u, v$ are non-negative lower semi-continuous functions with $f=u-v$ and $\|f\|_{D}=\|u+v\|_{\infty}$.

We first prove the theorem, then give several remarks. The proof requires the following two lemmas. (Throughout, $K$ is a given separable metric space.)

Lemma 3.6. Let $u, v$ be non-negative bounded lower semi-continuous functions defined on $K$. Then for all countable ordinals $\alpha$,

$$
\operatorname{osc}_{\alpha}(u-v) \leq \operatorname{osc}(u+v) \text {. }
$$

Proof. (52) trivially holds for $\alpha=0$. Let $\alpha$ be a countable ordinal and suppose (52) holds. Let $x \in K$. We first show

$$
\widetilde{\mathrm{OsC}}_{\alpha+1}(u-v)(x) \leq \operatorname{osc}(u+v)(x) \text {. }
$$

We may choose $\left(y_{n}\right)$ a sequence tending to $x$ so that

$$
\begin{aligned}
& \widetilde{\mathrm{osc}}_{\alpha+1}(u-v)(x) \\
& \quad=\lim _{n \rightarrow \infty}\left[\left|\left(u\left(y_{n}\right)-v\left(y_{n}\right)\right)-(u(x)-v(x))\right|+\operatorname{osc}_{\alpha}\left(u\left(y_{n}\right)-v\left(y_{n}\right)\right)\right] .
\end{aligned}
$$

Since $u, v$, and $\operatorname{osc}_{\alpha}(u-v)$ are bounded, we may assume without loss of generality that $\lim _{n \rightarrow \infty} u\left(y_{n}\right), \lim _{n \rightarrow \infty} v\left(y_{n}\right), \lim _{n \rightarrow \infty} \operatorname{osc}_{\alpha}\left(u\left(y_{n}\right)-v\left(y_{n}\right)\right)$, and $\lim _{n \rightarrow \infty} \operatorname{osc}\left(u\left(y_{n}\right)+v\left(y_{n}\right)\right)$ all exist. We then have, by (54) and the assumption that (52) holds, that

$$
\begin{aligned}
\widetilde{\operatorname{osc}}_{\alpha+1}(u-v)(x) \leq \lim _{n \rightarrow \infty}\left(\left|u\left(y_{n}\right)-u(x)\right|\right. & +\left|v\left(y_{n}\right)-v(x)\right| \\
& \left.+\operatorname{osc}_{\alpha}\left(u\left(y_{n}\right)+v\left(y_{n}\right)\right)\right) .
\end{aligned}
$$


We next observe that

$$
\lim _{n \rightarrow \infty}\left|u\left(y_{n}\right)-u(x)\right|+\left|v\left(y_{n}\right)-v(x)\right|=\lim _{n \rightarrow \infty}\left|u\left(y_{n}\right)+v\left(y_{n}\right)-u(x)-v(x)\right|
$$

Indeed, this follows immediately from the observation that, since $u$ is lower semi-continuous,

$$
\lim _{n \rightarrow \infty} u\left(y_{n}\right)-u(x) \geq 0
$$

whence $\lim _{n \rightarrow \infty}\left|u\left(y_{n}\right)-u(x)\right|=\lim _{n \rightarrow \infty} u\left(y_{n}\right)-u(x)$. Thus (56) holds since all the limits are the same, upon removing the absolute value signs, noting that $v$ and $u+v$ are also lower semi-continuous. Now (55), (56) yield that

$$
\begin{aligned}
\widetilde{\text { osc }}_{\alpha+1}(u-v)(x) & \leq \lim _{n \rightarrow \infty}\left|u\left(y_{n}\right)+v\left(y_{n}\right)-u(x)-v(x)\right|+\operatorname{osc}_{\alpha}\left(u\left(y_{n}\right)+v\left(y_{n}\right)\right) \\
& \leq \widetilde{\text { osc }}_{\alpha+1}(u(x)+v(x))=\operatorname{osc}(u(x)+v(x))
\end{aligned}
$$

(by Proposition 3.4 (d)).

Of course (53) is now established; but then since we have that $\widetilde{\text { osc }}_{\alpha+1}(u-v)$ $\leq \operatorname{osc}(u+v), \operatorname{osc}_{\alpha+1}(u-v)=U \widetilde{o s c}_{\alpha+1}(u-v) \leq U \operatorname{osc}(u+v)=\operatorname{osc}(u+v)$. Finally, suppose $\beta$ is a limit ordinal and (52) is established for all $\alpha<\beta$. But then immediately

$$
\widetilde{\operatorname{osc}}_{\beta}(u-v)=\sup _{\alpha<\beta} \operatorname{osc}_{\alpha}(u-v) \leq \operatorname{osc}(u+v),
$$

so again $\operatorname{osc}_{\beta}(u-v)=U \operatorname{osc}_{\beta}(u-v) \leq \operatorname{osc}(u+v)$. This completes the proof of the lemma.

Finally, we require the following known stability result (cf. [KL]), which we prove here for the sake of completeness. ( $w_{1}$ denotes the first uncountable ordinal.)

Lemma 3.7. Let $\left(\varphi_{\alpha}\right)_{\alpha<w_{1}}$ be a family of upper semi-continuous extended realvalued functions defined on $K$ so that $\varphi_{\alpha} \leq \varphi_{\beta}$ for all $\alpha \leq \beta$. Then there is $a$ countable ordinal $\alpha$ so that $\varphi_{\alpha}=\varphi_{\beta}$ for all $\beta>\alpha$.

Proof. Suppose not. Then by renumbering, we may assume that

$$
\varphi_{\alpha} \neq \varphi_{\alpha+1} \text { for all } \alpha<w_{1} \text {. }
$$

Now let $\mathscr{B}$ be a countable base for the open subsets of $K$. Fix $\alpha<w_{1}$; by (58), we may choose $x=x_{\alpha} \in K$ with $\varphi_{\alpha}(x)<\varphi_{\alpha+1}(x)$. Then by upper semi-continuity of $\varphi_{\alpha}$, choose $U_{\alpha} \in \mathscr{B}$ so that $x \in U_{\alpha}$ and

$$
\lambda_{\alpha} \stackrel{\mathrm{df}}{=} \sup \varphi_{\alpha}\left(U_{\alpha}\right)<\varphi_{\alpha+1}(x) \text {. }
$$

Since $w_{1}$ is uncountable, we may choose an uncountable subset $\Gamma$ of $w_{1}$ so that

$$
U_{\alpha}=U_{\beta} \stackrel{\text { df }}{=} U \text { for all } \alpha, \beta \in \Gamma .
$$

Finally, we claim that

$$
\lambda_{\alpha}<\lambda_{\beta} \text { if } \alpha<\beta, \alpha, \beta \in \Gamma \text {. }
$$


Indeed, fixing $\alpha<\beta$ in $\Gamma$ and letting $x=x_{\alpha}$ as above, we have that $\lambda_{\alpha}<$ $\varphi_{\alpha+1}(x) \leq \varphi_{\beta}(x) \leq \sup \varphi_{\beta}(U)=\lambda_{\beta}$. But of course since $\Gamma$ is uncountable, (61) is impossible.

Proof of Theorem 3.5. The first assertion follows immediately from the preceding lemma and Proposition 3.4(a). Now first assume $f$ is real valued. If $f$ is in $D(K)$, we may choose $u, v$ lower semi-continuous bounded non-negative functions with $f=u-v$, and then by Lemma 3.6, letting $\tau$ be as in the statement of the theorem, $\operatorname{osc}_{\tau} f \leq \operatorname{osc}(u+v)$, a bounded function. Now suppose conversely that osc $f$ is bounded, and let $\lambda, u$, and $v$ be as in the statement of Theorem 3.5. Then it's immediate that $f=u-v$ and $u, v$ are non-negative. But by Proposition 3.4(c), since osc $f=\operatorname{osc}_{\tau+1} f, \operatorname{osc}_{\tau} f \pm f$ are upper semi-continuous, which implies the lower semi-continuity of $u$ and $v$. Thus since $u$ and $v$ are bounded, it is proved that $f$ is in $D(K)$. Finally, for the norm identity, we first note (by (40)) that

$$
\|f\|_{D} \leq\|u+v\|_{\infty}=\left\|\lambda-\operatorname{osc}_{\tau} f\right\|_{\infty} \leq \lambda
$$

(the last inequality holds since $0 \leq \operatorname{osc}_{\tau} f \leq \lambda$ ). For the reverse inequality, let $\varepsilon>0$ and choose $g, h$ non-negative lower semi-continuous with $f=g-h$ and

$$
\|g+h\|_{\infty} \leq\|f\|_{D}+\varepsilon
$$

Now we have that

$$
\begin{aligned}
|f|+ & \text { osc }_{\tau} f=|g-h|+\operatorname{osc}_{\tau}(g-h) \\
& \leq|g-h|+\operatorname{osc}(g+h) \quad(\text { by Lemma 3.6) } \\
& =|g-h|+U(g+h)-(g+h)(\text { since } g+h \text { is lower semi-continuous) } \\
& \leq U(g+h)(\text { since }|g-h|-(g+h) \leq 0) .
\end{aligned}
$$

Hence

$$
\lambda=\left\||f|+\operatorname{osc}_{\tau} f\right\|_{\infty} \leq\|U(g+h)\|_{\infty}=\|g+h\|_{\infty} \leq\|f\|_{D}+\varepsilon .
$$

Since $\varepsilon>0$ is arbitrary, $\lambda \leq\|f\|_{D}$, so by (62), the theorem is established for real-valued $f$. Now suppose $f$ is complex-valued. Then it is easily established by transfinite induction that if $g=\operatorname{Re} f$ or $\operatorname{Im} f$, then

$$
\text { osc }_{\alpha} g \leq \text { osc }_{\alpha} f \text { for all ordinals } \alpha \text {. }
$$

Thus we obtain that $\operatorname{osc}_{\beta} g \leq \operatorname{osc}_{\beta} f=\operatorname{osc}_{\tau} f$ for all $\beta>\tau$ (where $\tau$ is as in the statement of the theorem). Hence if $\operatorname{osc}_{\tau} f$ is bounded and $\beta$ is such that $\operatorname{osc}_{\beta+1} g=\operatorname{osc}_{\beta} g$ for both $g=\operatorname{Re} f$ and $g=\operatorname{Im} f$, then $\operatorname{osc}_{\beta} \operatorname{Re} f$, $\operatorname{osc}_{\beta} \operatorname{Im} f$ are both bounded, whence $f$ is in $D(K)$ since its real and imaginary parts belong to $D(K)$. Of course if $f$ is in $D(K)$, then we trivially have that $\operatorname{Re} f, \operatorname{Im} f$ belong to $D(K)$, and then osc $_{\tau} f \leq \operatorname{osc}_{\tau} \operatorname{Re} f+\operatorname{osc}_{\tau} \operatorname{Im} f$ by Proposition 3.4b; thus $\operatorname{osc}_{\tau} f$ is bounded. This completes the proof of Theorem 3.5.

We proceed now with several complements and remarks concerning Theorem 3.5. 
Let $f: K \rightarrow \mathbb{C}$ be a general function. We define the $D$-index of $f$, denoted $i_{D}(f)$, to be the least ordinal $\alpha$ so that $\operatorname{osc}_{\alpha} f=\operatorname{osc}_{\alpha+1} f$. It is shown in [R5] that, for $f \in D[0,1], i_{D} f$ may be any countable ordinal (an analogous index and result were previously obtained in $[\mathrm{KL}])$.

Now letting $\alpha=i_{D} f$ and assuming $f \in D(K)$, equivalently by Theorem 3.5 that $f$ and osc $_{\alpha} f$ are bounded, we have by Proposition 3.4 that assuming $f$ is real valued, osc $f \pm f$ are both upper semi-continuous. It also follows that osc $\alpha+|f|$, osc $f+f^{+}$, and osc $_{\alpha} f+f^{-}$are all upper semi-continuous. Indeed, osc $_{\alpha} f+|f|=\max \left\{\right.$ osc $_{\alpha} f+f$, osc $\left._{\alpha} f-f\right\}$, and the max of two upper semi-continuous functions is again upper semi-continuous. But of course since $f^{+}=\frac{|f|+f}{2}, \operatorname{osc}_{\alpha} f+f^{+}=\frac{\left.\operatorname{osc}_{\alpha} f+|f|\right)+\left(\operatorname{osc}_{\alpha} f+f\right)}{2}$ is again upper semi-continuous, with a similar argument for $f^{-}$. Thus we also obtain $f$ as the difference of two non-negative upper semi-continuous functions, $u=\operatorname{osc}_{\alpha} f+f^{+}$and $v=$ osc $_{\alpha} f+f^{-}$, and again $\|u+v\|_{\infty}=\|f\|_{D}$.

We also note that, for $f \in D(K)$ complex-valued and $\alpha$ as above, we have

$$
\frac{1}{2}\|f\|_{D} \leq\left\||f|+\operatorname{osc}_{\alpha} f\right\|_{\infty} \leq 2\|f\|_{D} .
$$

(This follows easily from (64) and the validity of (51) for $\operatorname{Re} f, \operatorname{Im} f$, where $\tau=\max \left\{i_{D} \operatorname{Re} f, i_{D} \operatorname{Im} f\right\}$.)

Now for $f: K \rightarrow \mathbb{C}$ bounded, which is not in $D(K)$, we obtain from Theorem 3.5 that there is a countable ordinal $\alpha$ so that $\operatorname{osc}_{\alpha} f$ is unbounded. We define $i_{N D} f$, the non- $D$ index of $f$, to be the least ordinal $\alpha$ so that this happens. It is obvious that $\alpha$ must be a limit ordinal, for $\|$ osc $_{\alpha+1} f \|_{\infty} \leq$ $2\|f\|_{\infty}+\left\|\operatorname{osc}_{\alpha} f\right\|_{\infty}$ for any ordinal $\alpha$. It is proved in [R5] that in fact $i_{N D}(f)$ must be an ordinal of the form $w^{\beta}$ for some countable non-zero ordinal $\beta$, and moreover for each such $\beta$, there is a function $f:[0,1] \rightarrow[0,1]$ with $i_{N D}(f)=w^{\beta}$. It's also worth observing that if $K$ is a compact metric space, if $f \notin D(K), f$ is bounded, and $\alpha=i_{N D}(f)$, then osc $\alpha$ must assume the value $+\infty$. Thus for $K$ compact metric, we have that a bounded $f$ is in $D(K)$ if (and only if) osc $\alpha$ is real valued for all countable ordinals $\alpha$.

We conclude this section with a discussion of the above indices and some other indices and transfinite invariants for $D(K)$ introduced earlier in [KL].

Definition 3.2. Let $f: K \rightarrow \mathbb{R}$ be a given function and $\alpha$ be a countable ordinal. We define the $\alpha^{\text {th }}$ positive oscillation of $f, v_{\alpha} f$, by induction, as follows: set $v_{0} f \equiv 0$. Suppose $\beta>0$ is a countable ordinal, and $v_{\alpha} f$ has been defined for all $\alpha<\beta$. If $\beta=\alpha+1$ for some $\alpha$, define $\tilde{v}_{\beta} f$ by

$$
\tilde{v}_{\beta} f(x)=\varlimsup_{y \rightarrow x}\left(f(y)-f(x)+v_{\alpha} f(y)\right) \text { for all } x \in K .
$$

If $\beta$ is a limit ordinal, set $\tilde{v}_{\beta} f(x)=\sup _{\alpha<\beta} v_{\alpha} f(x)$ for all $x \in K$. Finally, let $v_{\beta} f=U \tilde{v}_{\beta} f$.

Of course the positive oscillations are defined exactly as in Definition 3.1, but we simply delete absolute values in the definition. The $v_{\alpha} f$ 's (with a different terminology and equivalent formulation) are given in [KL], where it is 
established (for $K$ compact metric, which is not essential to the argument) that again $f \in D(K)$ if and only if $v_{\alpha} f$ is uniformly bounded for all $\alpha$. Thus when the $v_{\alpha}(f)$ 's are bounded, choosing $\alpha$ so that $v_{\alpha}(f)=v_{\alpha+1}(f)$, and writing $f=u_{\alpha}-v_{\alpha}$, it follows (as shown in [KL]) that $u_{\alpha}$ is upper semi-continuous, and hence $f$ is in $D(K)$.

The following simple result gives the basic connection between the transfinite oscillations and transfinite positive oscillations.

Proposition 3.8. Let $f: K \rightarrow \mathbb{R}$ be a given function and $\alpha$ be a countable ordinal. Then

$$
v_{\alpha}(f) \leq \operatorname{osc}_{\alpha} f \leq v_{\alpha}(f)+v_{\alpha}(-f) .
$$

Proof. The first inequality follows immediately from the definitions and transfinite induction. For the second, suppose $\beta>0$ is such that the inequality is proved for all $\alpha<\beta$. If $\beta$ is a successor ordinal, say $\beta=\alpha+1$, given $x \in K$, we may choose a sequence $\left(x_{n}\right)$ in $K$ with $x_{n} \rightarrow x$ so that $\lim _{n \rightarrow \infty} f\left(x_{n}\right)$ and $\lim _{n \rightarrow \infty} \operatorname{osc}_{\alpha} f\left(x_{n}\right)$ both exist (as extended real numbers) and either

$$
\widetilde{\mathrm{osc}}_{\alpha+1} f(x)=\lim _{n \rightarrow \infty} f\left(x_{n}\right)-f(x)+\text { osc }_{\alpha} f\left(x_{n}\right)
$$

(with $\lim _{n \rightarrow \infty} f\left(x_{n}\right)-f(x) \geq 0$ ) or

$$
\widetilde{\mathrm{osc}}_{\alpha+1} f(x)=\lim _{n \rightarrow \infty} f(x)-f\left(x_{n}\right)+\operatorname{osc}_{\alpha} f\left(x_{n}\right)
$$

(with $\lim _{n \rightarrow \infty} f\left(x_{n}\right)-f(x) \leq 0$ ).

But in the first instance, we have (using (67)) that

$$
\begin{aligned}
\widetilde{\mathrm{osc}}_{\alpha+1} f(x) & \leq \lim _{n \rightarrow \infty} f\left(x_{n}\right)-f(x)+v_{\alpha} f\left(x_{n}\right)+v_{\alpha}(-f)\left(x_{n}\right) \\
& \leq \tilde{v}_{\alpha+1}(f)(x)+v_{\alpha}(-f)(x) \\
& \leq v_{\alpha+1}(f)(x)+v_{\alpha+1}(-f)(x)
\end{aligned}
$$

Again in the second case,

$$
\begin{aligned}
\widetilde{o s c}_{\alpha+1} f(x) & \leq \lim _{n \rightarrow \infty}(-f)\left(x_{n}\right)-(-f)(x)+v_{\alpha}(-f)\left(x_{n}\right)+v_{\alpha} f\left(x_{n}\right) \\
& \leq \tilde{v}_{\alpha+1}(-f)(x)+v_{\alpha} f(x) \\
& \leq v_{\alpha+1} f(x)+v_{\alpha+1}(-f)(x) .
\end{aligned}
$$

Thus, we have

$$
\widetilde{\mathrm{osc}}_{\beta} f \leq v_{\beta}(f)+v_{\beta}(-f) .
$$

If $\beta$ is a limit ordinal, then

$$
\begin{aligned}
\widetilde{\operatorname{osc}_{\beta} f} & =\sup _{\alpha<\beta} \operatorname{osc}_{\alpha} f \leq \sup _{\alpha<\beta} v_{\alpha}(f)+v_{\alpha}(-f) \\
& \leq \sup _{\alpha<\beta} v_{\alpha}(f)+\sup _{\alpha<\beta} v_{\alpha}(-f)=\tilde{v}_{\beta}(f)+\tilde{v}_{\beta}(-f) \leq v_{\beta}(f)+v_{\beta}(-f),
\end{aligned}
$$

i.e., again (69) holds. But then immediately

$$
\operatorname{osc}_{\beta} f=U \widetilde{\operatorname{osc}}_{\beta} f \leq U\left(v_{\beta}(f)+v_{\beta}(-f)\right)=v_{\beta}(f)+v_{\beta}(-f) .
$$


Remark. It is easily seen that if $X$ is a separable Banach space, $\Omega=B a\left(X^{*}\right)$ endowed with the weak* topology, $x^{* *} \in X^{* *}$, and $f=x^{* *} \mid \Omega$, then, for the real scalars case,

$$
v_{\alpha}(f)(-w)=v_{\alpha}(-f)(w) \text { for all } w \in \Omega \text {. }
$$

Hence by $3.8,\left(\operatorname{osc}_{\alpha} f\right)(w) \leq 2 \max _{ \pm}\left(v_{\alpha} f\right)( \pm w)$ for all $w \in \Omega$, whence $\left\|\operatorname{osc}_{\alpha} f\right\|_{\infty} \leq 2\left\|v_{\alpha} f\right\|_{\infty}$. It also then follows for the case of complex scalars that $\left\|\operatorname{osc}_{\alpha} f\right\|_{\infty} \leq 4\left\|v_{\alpha} \operatorname{Re} f\right\|_{\infty}$. That is, the positive transfinite oscillations have essentially the same growth as the transfinite oscillations, in the case of our main application here.

To prove our main result, Theorem 1.1, we formulate a method for computing $v_{\alpha+1}(\varphi)$ in terms of $\tilde{v}_{\alpha}(\varphi)$ and $\tilde{v}_{1}(\varphi)$.

Lemma 3.9. Let $\alpha$ be a countable ordinal, $x \in K, \varphi: K \rightarrow \mathbb{R}$ be a given function, and assume $0<v_{\alpha}(\varphi)(x)<v_{\alpha+1}(\varphi)(x) \stackrel{\mathrm{df}}{=} \beta<\infty$; let $U$ be an open neighborhood of $x$ and $\eta>0$ be given. There exist positive numbers $\underline{\lambda}$ and $\delta$ and $x_{1} \in U$ so that

(1) $(1-\eta) \beta<\underline{\lambda}+\delta<(1+\eta) \beta$,

(2) $x_{1} \in \bar{L}$ where $L=\left\{y: \underline{\lambda} \leq v_{\alpha}(\varphi)(y)<(1+\eta) \beta-\delta\right\}$,

(3) $\varlimsup_{\lim _{y \rightarrow x_{1}} ; y \in L}\left(\varphi(y)-\varphi\left(x_{1}\right)\right)=\delta$.

Proof. This argument is just at the definitional level, but we give all the tedious details, to be thorough.

For convenience, define $V_{\lambda}$ for $\lambda>0$ by

$$
V_{\lambda}=\left\{y: v_{\alpha}(\varphi)(y) \geq \lambda\right\} .
$$

Of course $V_{\lambda}$ is closed, by the upper semi-continuity of $v_{\alpha}(\varphi)$, but we have no need of this fact. Next we observe

$$
\text { if } y \in V_{\lambda}, \text { then } \lambda+\tilde{v}_{1}\left(\varphi \mid V_{\lambda}\right)(y) \leq \tilde{v}_{\alpha+1}(\varphi)(y) .
$$

We note in passing that (71) yields immediately that for all $y \in V_{\lambda}, \lambda+$ $v_{1}\left(\varphi \mid V_{\lambda}\right)(y) \leq v_{\alpha+1}(\varphi)(y)$. To see (71), choose $\left(y_{n}\right) \in V_{\lambda}$ with $y_{n} \rightarrow y$ and

$$
\lim _{n \rightarrow \infty} \varphi\left(y_{n}\right)-\varphi(y)=\tilde{v}_{1}\left(\varphi \mid V_{\lambda}\right)(y) \text {. }
$$

But then

$$
\begin{aligned}
\tilde{v}_{\alpha+1}(\varphi)(y) & \geq \lim _{n \rightarrow \infty} \varphi\left(y_{n}\right)-\varphi(y)+v_{\alpha}(\varphi)\left(y_{n}\right) \\
& \geq \tilde{v}_{1}\left(\varphi \mid V_{\lambda}\right)(y)+\lambda .
\end{aligned}
$$

Thus (71) follows directly from the definitions.

Next, for convenience, by taking $\eta$ small enough, we may assume that

$$
v_{\alpha}(\varphi)(x)<(1-\eta) \beta \text {. }
$$

By upper semi-continuity of $v_{\alpha}(\varphi)$ and $v_{\alpha+1}(\varphi)$, choose $\mathscr{V}$ open with $x \in$ $\mathscr{V} \subset \mathscr{U}$ so that

$$
v_{\alpha}(\varphi)(v)<(1-\eta) \beta \text { and } v_{\alpha+1}(\varphi)(v)<\left(1+\frac{\eta}{2}\right) \beta \text { for all } v \in \mathscr{V} .
$$


Now choose $x_{1} \in \mathscr{V}$ with

$$
(1-\eta) \beta<\tilde{v}_{\alpha+1}(\varphi)\left(x_{1}\right) .
$$

Next choose $\left(y_{n}\right)$ a sequence with $y_{n} \rightarrow x_{1}$ and

$$
\lim _{n \rightarrow \infty} \varphi\left(y_{n}\right)-\varphi\left(x_{1}\right)+v_{\alpha}(\varphi)\left(y_{n}\right)=\tilde{v}_{\alpha+1}(\varphi)\left(x_{1}\right) \text {. }
$$

By passing to a subsequence, we may assume

$$
\lim _{n \rightarrow \infty} \varphi\left(y_{n}\right)-\varphi\left(x_{1}\right) \stackrel{\text { df }}{=} \kappa \text { and } \lim _{n \rightarrow \infty} v_{\alpha}(\varphi)\left(y_{n}\right) \stackrel{\text { df }}{=} \lambda \text { both exist. }
$$

Now $\kappa \leq \tilde{v}_{1}(\varphi)\left(x_{1}\right) \leq \tilde{v}_{\alpha}(\varphi)\left(x_{1}\right)$ and $\lambda \leq v_{\alpha}(\varphi)\left(x_{1}\right)$, and $v_{\alpha}(\varphi)\left(x_{1}\right)<$ $(1-\eta) \beta$ by $(74)$, yet

$$
(1-\eta) \beta<\lambda+\kappa=\tilde{v}_{\alpha+1}(\varphi)\left(x_{1}\right) \text { by }(75) .
$$

Hence both $\lambda$ and $\kappa$ are positive. Now let $0<\underline{\lambda}<\lambda$ be such that

$$
\underline{\lambda}+\kappa>(1-\eta) \beta \text { and } \lambda-\underline{\lambda}<\frac{\eta}{2} \beta \text {. }
$$

Finally, set $\delta=\tilde{v}_{1}\left(\varphi \mid V_{\underline{\lambda}}\right)\left(x_{1}\right)$. Since the definition of $\lambda$ now yields that $v_{\alpha}(\varphi)\left(y_{n}\right)$ $>\underline{\lambda}$ for all $n$ sufficiently large, we have that $\kappa \leq \delta$, and hence $\delta>0$, and moreover

$$
\begin{aligned}
\underline{\lambda}+\delta & \leq \tilde{v}_{\alpha+1}(\varphi)\left(x_{1}\right) & & \text { by (71) } \\
& \leq v_{\alpha+1}(\varphi)\left(x_{1}\right)<\left(1+\frac{\eta}{2}\right) \beta & & \text { by (74) }
\end{aligned}
$$

hence (1) of 3.9 holds (using (79) and (80)). Again, we have, since $\delta+\underline{\lambda}<$ $\left(1+\frac{\eta}{2}\right) \beta$ and $\lambda<\underline{\lambda}+\frac{\eta}{2} \beta$ by (79), that

$$
\delta+v_{\alpha}\left(\varphi\left(y_{n}\right)\right)<\delta+\underline{\lambda}+\frac{\eta}{2} \beta<(1+\eta) \beta \text { for all } n \text { sufficiently large, }
$$

whence (2) of 3.9 holds, since $y_{n} \in L$ for all such $n$. To see (3), simply choose $\left(z_{n}\right) \in V_{\underline{\lambda}}$ with $z_{n} \rightarrow x_{1}$ and

$$
\lim _{n \rightarrow \infty} \varphi\left(z_{n}\right)-\varphi\left(x_{1}\right)=\delta
$$

But then

$$
\begin{gathered}
\varlimsup_{n \rightarrow \infty} \varphi\left(z_{n}\right)-\varphi\left(x_{1}\right)+v_{\alpha}(\varphi)\left(z_{n}\right)=\lim _{n \rightarrow \infty} \varphi\left(z_{n}\right)-\varphi\left(z_{1}\right)+\varlimsup_{n \rightarrow \infty} v_{\alpha}(\varphi)\left(z_{n}\right) \\
=\delta+\varlimsup_{n \rightarrow \infty} v_{\alpha}(\varphi)\left(z_{n}\right) \leq \tilde{v}_{\alpha+1}(\varphi)\left(x_{1}\right)<(1+\eta) \beta .
\end{gathered}
$$

Thus (82) yields that

$$
v_{\alpha} \varphi\left(z_{n}\right)<(1+\eta) \beta-\delta \text { for all } n \text { sufficiently large; }
$$

i.e., $z_{n} \in L$ for all such $n$, and so

$$
\begin{aligned}
\delta & \leq \varlimsup_{y \rightarrow x_{1}}(\varphi \mid L)(y)-\varphi\left(x_{1}\right) \leq \varlimsup_{y \rightarrow x_{1}}\left(\varphi \mid V_{\underline{\lambda}}\right)(y)-\varphi\left(x_{1}\right) \\
& =\tilde{v}_{1}\left(\varphi \mid V_{\underline{\lambda}}\right)\left(x_{1}\right)=\delta,
\end{aligned}
$$

proving (3). 


\section{Proof of the MAIN THEOREM}

The following result is the central concern of this section; it quickly leads to a proof of our main result, via the preceding development.

Theorem 4.1. Let $\alpha \geq 1$ be a countable ordinal, $K$ be a separable metric space, $f: K \rightarrow \mathbb{C}$, and $\left(f_{j}\right)$ be a uniformly bounded sequence of complex-valued continuous functions on $K$ with $f_{j} \rightarrow f$ pointwise. Also let $x \in K$ and assume $0<v_{\alpha}(\operatorname{Re} f)(x) \stackrel{\text { df }}{=} \lambda<\infty ;$ let $0<\eta<1$. There exists $\left(b_{j}\right)$, a subsequence of $\left(f_{j}\right)$, so that setting $e_{1}=b_{1}, e_{j}=b_{j}-b_{j-1}$ for all $j>1$, then given $1=m_{1}<m_{2}<\cdots$ an infinite sequence of indices, there exist $a t$ in $K$ and an integer $k$ with
(1) $\sum_{j=1}^{k} \operatorname{Re} e_{m_{2 j}}(t)>(1-\eta) \lambda$,
(2) $\operatorname{Re} e_{m_{2 j}}(t)>0$ for all $1 \leq j \leq k$,
(3) $\sum_{i \notin\left\{m_{1}, m_{2}, \ldots\right\}}\left|e_{i}(t)\right|<\eta \lambda$.

We first deduce Theorem 1.8 from (4.1) and our previous results. Let $f$ be as in Theorem 1.8, and $\beta=i_{N D}(f)$. Now we could replace $f$ by $\tilde{f}$, where

$$
\tilde{f}(\mu)=\int f d \mu \text { for all } \mu \in \tilde{K} \stackrel{\mathrm{df}}{=} B a\left(C(K)^{*}\right)=B a(M(K)),
$$

$M(K)$ the finite complex Borel measures on $K$. Since $\left\|\operatorname{osc}_{\alpha}(\tilde{f})\right\|_{\infty} \leq$ $4\left\|v_{\alpha} \operatorname{Re} \tilde{f}\right\|_{\infty}$ for all countable ordinals $\alpha$, by the remark following the proof of Proposition 3.8, we would then have directly that $v_{\beta}(\varphi)$ is unbounded but $v_{\alpha}(\varphi)$ is bounded for all $\alpha<\beta$. We prefer to see this "directly", by a "realvariables" argument. Indeed, since $\operatorname{osc}_{\beta}(f) \leq \operatorname{osc}_{\beta}(\operatorname{Re} f)+\operatorname{osc}_{\beta}(\operatorname{Im} f)$, either $\operatorname{osc}_{\beta}(\operatorname{Re} f)$ or $\operatorname{osc}_{\beta}(\operatorname{Im} f)$ must be unbounded, so by replacing $f$ by if and $\left(f_{n}\right)$ by $i f_{n}$ for all $n$ if necessary, we may assume without loss of generality that $\operatorname{osc}_{\beta}(\operatorname{Re} f)$ is unbounded and hence $\beta=i_{N D}(\operatorname{Re} f)$ since, by definition, $\operatorname{osc}_{\alpha}(f)$ is bounded for all $\alpha<\beta$. Again, since $\operatorname{osc}_{\beta}(\operatorname{Re} f) \leq$ $v_{\beta}(\operatorname{Re} f)+v_{\beta} \operatorname{Re}(-f)$ by Proposition 3.8, we may assume, by replacing $f$ by $-f$ and $f_{n}$ by $-f_{n}$ for all $n$ if necessary, that $v_{\beta}(\operatorname{Re} f)$ is unbounded. Now as noted in the preceding section, $\beta$ is a limit ordinal, and we thus have (since $\tilde{v}_{\beta}(\operatorname{Re} f)$ is also trivially unbounded) that

$$
v_{\alpha}(\operatorname{Re} f) \text { is bounded for all } \alpha<\beta \text { and } \sup _{\alpha<\beta}\left\|v_{\alpha}(\operatorname{Re} f)\right\|_{\infty}=\infty .
$$

Next, by Proposition 2.2, we may assume without loss of generality that $\left(f_{j}\right)$ is an (s)-sequence in the Banach space $C(K)$, since $\left(f_{j}\right)$ is non-trivial weakCauchy in $C(K)$. Then by P1 in the proof of Lemma 2.8, we may choose $1 \leq \tau<\infty$ so that for all subsequences $\left(f_{j}^{\prime}\right)$ of $\left(f_{j}\right)$,

the biorthogonal functionals for $\left(f_{j}^{\prime}-f_{j-1}^{\prime}\right)$ all have norm at most $\tau$, and also $\left\|f_{j}^{\prime}-f_{j-1}^{\prime}\right\| \leq \tau$ for all $j$. 
To prove that $\left(f_{j}\right)$ has an (s.s.)-subsequence, we apply Lemma 2.8. Thus, let $\varepsilon>0$ and $\left(f_{j}^{\prime}\right)$ an arbitrary subsequence of $\left(f_{j}\right)$ be given. By (83), choose $x \in K$ and $\alpha<\beta$ so that

$$
\lambda \stackrel{\mathrm{df}}{=} v_{\alpha}(\operatorname{Re} f)(x)>\frac{2}{\varepsilon} .
$$

Now let $0<\eta<1$, to be determined later, and choose by Theorem $4.1 \mathrm{a}$ subsequence $\left(b_{j}\right)$ of $\left(f_{j}^{\prime}\right)$ satisfying its conclusion. We shall show for appropriate $0<\eta<1$ that the difference sequence $\left(e_{j}\right)$ of $\left(b_{j}\right)$ is an $\varepsilon$-(c.c.) sequence. Suppose that this were not the case. Then we could choose scalars $\left(c_{j}\right)$ so that

$$
\begin{aligned}
& \left\|\sum_{j=1}^{n} c_{j} e_{j}\right\| \leq 1 \text { for all } n, \\
& c_{j}=0 \text { for infinitely many } j \text { (with } c_{1}=0 \text { ), } \\
& \left|c_{j}\right|>\varepsilon \text { for infinitely many } j .
\end{aligned}
$$

Now (86iii) means we may choose $M$ an infinite subset of $N$ and numbers $\rho_{j}, \theta_{j}$ with $\rho_{j}$ real, $\rho_{j}>\varepsilon$, and $\theta_{j}$ complex, $\left|\theta_{j}\right|=1$, and $c_{j}=\rho_{j} \theta_{j}$ for all $j \in M$. But then without loss of generality, we may assume the $\theta_{j}$ 's converge to $\theta$ say (for $j$ in $M$ ). By replacing $c_{j}$ by $\bar{\theta} c_{j}$ for all $j,(86 \mathrm{i}$-iii) are all unchanged, and we now have without loss of generality that $\theta=1$. Since $\left|c_{j}\right| \leq \tau$ for all $j$ by (84) and (86i), and $\lim _{j \rightarrow \infty, j \in M} \frac{c_{j}}{\left|c_{j}\right|}=1$, we may choose $1=m_{1}<m_{2}<\cdots$ so that for all $j$,

$$
\left\{\begin{array}{l}
c_{m_{2 j-1}}=0 \text { and } c_{m_{2 j}}=r_{j}+\delta_{j} \\
\text { where } r_{j} \text { is real, } r_{j}>\varepsilon, \text { and }\left|\delta_{j}\right|<\frac{\eta}{2^{j}} .
\end{array}\right.
$$

At last, choose $t$ in $K$ and $k$ an integer satisfying the conclusion of Theorem 4.1 for $\left(m_{j}\right)_{j=1}^{\infty}$. Then we have

$$
\sum_{i=1}^{m_{2 k}} c_{i} e_{i}(t)=\sum_{i=1}^{k} r_{i} e_{m_{2 i}}(t)+\sum_{i=1}^{k} \delta_{i} e_{m_{2 i}}(t)+\sum_{\substack{i \neq m_{j} \text { any } j \\ i \leq m_{2 k}}} c_{i} e_{i}(t) .
$$

Hence

$$
\begin{aligned}
\left\|\sum_{i=1}^{m_{2 k}} c_{i} e_{i}\right\| & \geq \sum_{i=1}^{k} r_{i} \operatorname{Re} e_{m_{2 i}}(t)-\sum_{i=1}^{k}\left|\delta_{i}\right|\left\|e_{m_{2 i}}\right\|-\sum_{i \neq m_{j} \text { any } j}\left|c_{i}\right|\left|e_{i}(t)\right| \\
& \geq \varepsilon(1-\eta) \lambda-\eta \tau-\tau \eta \lambda
\end{aligned}
$$

by (1)-(3) of Theorem 4.1 and the fact that $\left|c_{i}\right| \leq \tau$ for all $i$. Now $0<\eta<1$ was arbitrary, so assume

$$
\eta<\left(2+\tau+\frac{2 \tau}{\varepsilon}\right)^{-1}
$$


Then by (85), we obtain

$$
\varepsilon(1-\eta) \lambda-\eta \tau-\tau \eta \lambda>2-2 \eta-\tau \eta-\frac{2 \tau \eta}{\varepsilon}>1
$$

Thus $\left\|\sum_{i=1}^{m_{2 k}} c_{i} e_{i}\right\|>1$, contradicting (86i).

We now formulate a "real-variables" result, Theorem 4.2 which yields Theorem 4.1; we show 4.2 implies 4.1 , and then set about the remaining crucial work needed to establish 4.2 .

Theorem 4.2. Let $\left(f_{j}\right)$ be a uniformly bounded sequence of complex-valued bounded continuous functions defined on $K$, a separable metric space, converging pointwise to a function $f$. Let $\alpha$ be a countable ordinal, and $x \in K$ be given with $0<v_{\alpha}(\varphi)(x) \stackrel{\text { df }}{=} \lambda<\infty$ where $\varphi=\operatorname{Re} f$. Let $\mathscr{U}$ be an open neighborhood of $x$, and $0<\eta<1$ be given. There exists $\left(b_{j}\right)$, a subsequence of $\left(f_{j}\right)$ with the following properties: Given $1=m_{1}<m_{2}<\cdots$ an infinite sequence of integers, there exist $k$, points $x_{1}, \ldots, x_{2 k-1}, x_{2 k} \stackrel{\mathrm{df}}{=} t$ in $\mathscr{U}$, and positive numbers $\delta_{1}, \ldots, \delta_{k}$ so that:

(1) $\varphi\left(x_{2 j}\right)-\varphi\left(x_{2 j-1}\right)>(1-\eta) \delta_{j}$ for all $1 \leq j \leq k$,

(2) $(1+\eta) \lambda>\sum_{j=1}^{k} \delta_{j}>(1-\eta) \lambda$,

(3) $\sum_{m_{j} \leq i<m_{j+1}}\left|b_{i}(t)-f\left(x_{j}\right)\right|<\eta \delta_{\left[\frac{i+1}{2}\right]}$ for all $1 \leq j \leq 2 k-1$,

(4) $\sum_{i \geq m_{2 k}}\left|b_{i}(t)-f(t)\right|<\eta \delta_{k}$.

Remarks. 1. It is evident that if $\left(b_{i}\right)$ satisfies the conclusion of 4.2 , so does any subsequence $\left(b_{i}^{\prime}\right)$ of $\left(b_{i}\right)$. (The convenient anchoring condition $m_{1}=1$ is really inessential, thanks to (3) for $j=1$.)

2. The proof shows that $x_{1}, \delta_{1}$ may be chosen independently of $\left(m_{j}\right)$.

3. The subsequence $\left(b_{i}\right)$ satisfying the conclusion of Theorem 4.2 really yields a "stopping time" on the set of infinite-subsets of $N ;\left(m_{1}, m_{2}, \ldots\right) \rightarrow$ $\left(m_{1}, \ldots, m_{k}\right)$. The proof yields that the rank of this stopping time depends only on $\alpha$. For example, when $\alpha<w$, the proof gives that we may simply take $k=\alpha$, and again when $\alpha=w$, we may take $k$ finite (depending on the given $\eta$ ).

We now give the deduction of Theorem 4.1 from Theorem 4.2. Let $\eta>0$, $\lambda, x$, etc. be as in the statement of Theorem 4.1. Now let $0<\bar{\eta}<\frac{1}{3}$ also satisfy the inequalities

$$
(1-3 \bar{\eta})(1-\bar{\eta}) \geq 1-\eta \text { and } 4 \bar{\eta}(1+\bar{\eta}) \leq \eta \text {. }
$$

Now choose $\left(b_{j}\right)$ a subsequence of $\left(f_{j}\right)$ satisfying the conclusion of Theorem 4.2 for " $\eta "=\bar{\eta}$. We claim $\left(b_{j}\right)$ satisfies the conclusion of Theorem 4.1. Let $1=m_{1}<m_{2}<\cdots$ be given, and choose $k$, points $x_{1}, \ldots, x_{2 k} \stackrel{\text { df }}{=} t$ in $K$, and positive numbers $\delta_{1}, \ldots, \delta_{k}$ satisfying (1)-(4) of Theorem 4.2. Now it follows by (3) that for each $j, 1 \leq j \leq k$,

$$
\left|b_{m_{2 j}}(t)-f\left(x_{2 j}\right)\right|<\bar{\eta} \delta_{j} \text { and }\left|b_{m_{2 j-1}}(t)-f\left(x_{2 j-1}\right)\right|<\bar{\eta} \delta_{j} .
$$


Hence

$$
\begin{aligned}
\operatorname{Re} e_{m_{2 j}}(t) & =\operatorname{Re} b_{m_{2 j}}(t)-\operatorname{Re} b_{m_{2 j-1}}(t) \\
& >\varphi\left(x_{2 j}\right)-\varphi\left(x_{2 j-1}\right)-2 \bar{\eta} \delta_{j} \\
& >(1-3 \bar{\eta}) \delta_{j} \text { by }(1) \text { of } 4.2 .
\end{aligned}
$$

Thus (2) of 4.1 is verified. Next,

$$
\begin{aligned}
\sum_{j=1}^{k} \operatorname{Re} e_{m_{2 j}}(t) & >(1-3 \bar{\eta}) \sum_{j=1}^{k} \delta_{j} \\
& >(1-3 \bar{\eta})(1-\bar{\eta}) \lambda \text { by }(2) \text { of } 4.2 \\
& \geq(1-\eta) \lambda \text { by }(90) .
\end{aligned}
$$

Thus (1) of 4.1 is proved, and it remains to prove (3). Now fix $j$ and suppose $m_{j}<i<m_{j+1}$. Then

$$
\left|e_{i}(t)\right|=\left|b_{i}(t)-b_{i-1}(t)\right| \leq\left|b_{i}(t)-f\left(x_{j}\right)\right|+\left|b_{i-1}(t)-f\left(x_{j}\right)\right| .
$$

Hence

$$
\sum_{m_{j}<i<m_{j+1}}\left|e_{i}(t)\right| \leq 2 \sum_{m_{j} \leq i<m_{j+1}}\left|b_{i}(t)-f\left(x_{j}\right)\right|<2 \bar{\eta} \delta_{\left[\frac{i+1}{2}\right]}
$$

(the last inequality holds by (3) of 4.2). Thus

$$
\sum_{j=1}^{2 k-1} \sum_{m_{j}<i<m_{j+1}}\left|e_{i}(t)\right|<4 \bar{\eta} \sum_{j=1}^{k-1} \delta_{j}+2 \bar{\eta} \delta_{k}
$$

But again using (93),

$$
\sum_{i>m_{2 k}}\left|e_{i}(t)\right| \leq 2 \sum_{i \geq m_{2 k}}\left|b_{i}(t)-f\left(x_{j}\right)\right|<2 \bar{\eta} \delta_{k}
$$

(the last inequality holds by (4) of 4.2). Combining (95) and (96), we have

$$
\begin{gathered}
\sum_{i \notin\left\{m_{1}, m_{2}, \ldots\right\}}\left|e_{i}(t)\right|<4 \bar{\eta} \sum_{j=1}^{k} \delta_{j}<4 \bar{\eta}(1+\bar{\eta}) \lambda \text { by }(2) \text { of } 4.2 \\
\leq \eta \lambda \text { by }(90) .
\end{gathered}
$$

Thus (3) of 4.1 holds, completing the proof.

We now deal with the proof of Theorem 4.2. Throughout, we let $K,\left(f_{j}\right)$, and $f$ be as in $4.2 ; \varphi=\operatorname{Re} f$. We shall prove the result by induction on $\alpha$. The following lemma easily yields the case $\alpha=1$ and will be crucial in the general inductive step.

Lemma 4.3. Let $x_{1} \in K, L$ a subset of $K$ with $x_{1} \in \bar{L}, \delta \stackrel{\mathrm{df}}{=} \overline{\lim }_{y \rightarrow x_{1}, y \in L} \varphi(y)-$ $\varphi\left(x_{1}\right)>0,1>\eta>0$, and $\mathscr{U}$ an open neighborhood of $x_{1}$ be given. There exists $\left(b_{j}\right)$, a subsequence of $\left(f_{j}\right)$ so that given any $m>1$, there exists an $x_{2} \in \mathscr{U} \cap L$ with

(1) $\varphi\left(x_{2}\right)-\varphi\left(x_{1}\right)>(1-\eta) \delta$ 
(2) $\sum_{1<i<m}\left|b_{i}\left(x_{2}\right)-f\left(x_{1}\right)\right|<\eta \delta$,

(3) $\sum_{i \geq m}\left|b_{i}\left(x_{2}\right)-f\left(x_{2}\right)\right|<\eta \delta$.

4.3 immediately yields Theorem 4.2 for $\alpha=1$. Indeed, let $\lambda=v_{1}(\varphi)(x)>$ $0, \mathscr{U}$ an open neighborhood of $x$, and $\eta>0$ be given. Choose $x_{1} \in \mathscr{U}$ with

$$
(1-\eta) \lambda<\delta<(1+\eta) \lambda \text { where } \delta=\varlimsup_{y \rightarrow x_{1}} \varphi(y)-\varphi\left(x_{1}\right) .
$$

Then if $\left(b_{j}\right)$ satisfies the conclusion of 4.3 , it satisfies the conclusion of Theorem 4.2, for $k=1$.

We prove 4.3 by constructing a sequence of integers $n_{1}<n_{2}<\cdots$ and $M_{0}, M_{1}, M_{2}, \ldots$ infinite subsets of $N$, satisfying certain properties. Then we show $\left(b_{i}\right)=\left(f_{n_{i}}\right)$ works. For $i$ an integer and $M$ an infinite subset of $N$, $i<M$ means $i<\min M$.

We first construct $M_{0}$ and $n_{1}$; then we specify the general construction in a sublemma.

Since $f_{j} \rightarrow f$ point-wise, choose $M_{0}$ infinite with

$$
\sum_{j \in M_{0}}\left|f_{j}\left(x_{1}\right)-f\left(x_{1}\right)\right|<\eta \delta .
$$

Then let $n_{1}$ equal the least element of $M_{0}$.

Sub-Lemma 1. There exist positive integers $n_{2}, n_{3}, \ldots$ and infinite subsets of $N, M_{1}, M_{2}, \ldots$ so that, for all $s \geq 1$,

$$
n_{1}<n_{2}<\cdots<n_{s}<M_{s}
$$

(100) $\quad M_{s} \subset M_{s-1}$;

(101) $\quad n_{s}$ is the least element of $M_{s-1}$;

(102) there exists an $x_{2} \in \mathscr{U} \cap L$ with

(i)

$$
\begin{array}{ll}
\text { (i) } & \sum_{1 \leq i \leq s}\left|f_{n_{i}}\left(x_{2}\right)-f\left(x_{1}\right)\right|<\eta \delta, \\
\text { (ii) } & \varphi\left(x_{2}\right)-\varphi\left(x_{1}\right)>(1-\eta) \delta, \\
\text { (iii) } & \sum_{i \in M_{s}}\left|f_{i}\left(x_{2}\right)-f\left(x_{2}\right)\right|<\eta \delta .
\end{array}
$$

Sub-Lemma 1 easily yields Lemma 4.3 ; i.e., then $\left(b_{i}\right) \stackrel{\text { df }}{=}\left(f_{n_{i}}\right)$ works. For let $1<m$ be given, and let $s=m-1$. Now choose $x_{2} \in \mathscr{U}$ satisfying (102). Then (1) and (2) of 4.3 follow immediately from (102ii) and (102i), while (3) holds by (102iii), since (100) and (101) yield that $\left\{n_{s+1}, n_{s+2}, \ldots\right\} \subset M_{s}$.

Proof of Sub-Lemma 1. We first complete the case $s=1$; i.e., we construct $x_{2} \in \mathscr{U} \cap L$ and $M_{1}$. Now (98) yields that $\left|f_{n_{1}}\left(x_{1}\right)-f\left(x_{1}\right)\right|<\eta \delta$, so by the continuity of $f_{n_{1}}$ we may choose an open $\mathscr{V} \subset \mathscr{U}$ with $x_{1} \in \mathscr{V}$ so that

$$
\left|f_{n_{1}}(t)-f\left(x_{1}\right)\right|<\eta \delta \text { for all } t \in \mathscr{V} .
$$


Now by the definition of $\delta$, choose $x_{2} \in \mathscr{V} \cap L$ satisfying (102ii); then (102i) holds for $s=1$ by (103). Finally, since $f_{j}\left(x_{2}\right) \rightarrow f\left(x_{2}\right)$, choose $M_{1}$ infinite with $n_{1}<M_{1} \subset M_{0}$, satisfying (102iii).

Now let $s \geq 1$ and suppose $n_{1}, \ldots, n_{s}$ and $M_{1}, \ldots, M_{s}$ have been constructed satisfying (99)-(102). Let $n_{s+1}$ be the least element of $M_{s}$. Since $\left\{n_{1}, \ldots, n_{s+1}\right\} \subset M_{0}$, we have by (98) that

$$
\sum_{i=1}^{s+1}\left|f_{n_{i}}\left(x_{1}\right)-f\left(x_{1}\right)\right|<\eta \delta .
$$

Thus by the continuity of $f_{n_{1}}, \ldots, f_{n_{s+1}}$, we may choose $\mathscr{V}$ an open neighborhood of $x_{1}$ with $\mathscr{V} \subset \mathscr{U}$ so that

$$
\sum_{i=1}^{s+1}\left|f_{n_{i}}(t)-f\left(x_{1}\right)\right|<\eta \delta \text { for all } t \in \mathscr{V} .
$$

Now by the definition of $\delta$, choose $x_{2} \in \mathscr{V} \cap L$ satisfying (102ii). Then (102i) holds for " $s$ " $=s+1$, by (105). Finally, since $f_{j}\left(x_{2}\right) \rightarrow f\left(x_{2}\right)$, again choose $M_{s+1} \subset M_{s} \sim\left\{n_{s+1}\right\}$ infinite so that (102iii) holds for " $s$ " $=s+1$. This completes the proof of Sub-Lemma 1 and hence of Lemma 4.3.

We now proceed with the main inductive step in the proof of Theorem 4.2; namely we let $\alpha \geq 1$, assume the result established for $\alpha$, and prove it for $\alpha+1$. Thus we fix $x \in K$ satisfying

$$
0<v_{\alpha+1}(\varphi)(x) \stackrel{\text { df }}{=} \beta<\infty
$$

and let $\mathscr{U}$ be a given open neighborhood of $x$. Now let $0<\underline{\eta}<1$; we prove the $\alpha+1$-case for " $\eta$ " $=\underline{\eta}$. Evidently we may assume $0<\bar{v}_{\alpha}(\varphi)(x)<$ $v_{\alpha+1}(\varphi)(x)$, or there is nothing to prove. Indeed if $v_{\alpha}(\varphi)(x)=v_{\alpha+1}(\varphi)(x)$, the result already follows by the case for $\alpha$. Note that $v_{\alpha}(\varphi)(x)=0$ is impossible, for otherwise $v_{1}(\varphi)(x)=0$, but it's easily seen that $v_{\alpha+1}(\varphi) \leq v_{1}(\varphi)+v_{\alpha}(\varphi)$, whence $v_{\alpha+1} \varphi(x)=0$, a contradiction.

Now let $0<\eta<1$ be small, to be determined later. By Lemma 3.9, we may choose positive numbers $\underline{\lambda}$ and $\delta$, and $x_{1} \in \mathscr{U}$ satisfying (1)-(3) of 3.9, where $L$ is as in (2) of 3.9. Now using Lemma 4.3 and passing to a subsequence of $\left(f_{j}\right)$, we may assume without loss of generality that $\left(f_{j}\right)$ itself satisfies the conclusion of 4.3. That is, we have: given any $m>1$, there exists an $x_{2} \in \mathscr{U}$ with

$$
\begin{aligned}
& \left.\underline{\lambda} \leq v_{\alpha}(\varphi)\left(x_{2}\right)<(1+\eta) \beta-\delta \quad \text { (i.e., } x_{2} \in L\right), \\
& \varphi\left(x_{2}\right)-\varphi\left(x_{1}\right)>(1-\eta) \delta \\
& \sum_{1 \leq i<m}\left|f_{i}\left(x_{2}\right)-f\left(x_{1}\right)\right|<\eta \delta \\
& \sum_{i \geq m}\left|f_{i}\left(x_{2}\right)-f\left(x_{2}\right)\right|<\eta \delta .
\end{aligned}
$$

Now as in the proof of Lemma 4.3 we shall construct $n_{1}<n_{2}<\cdots$ and infinite sets $M_{1}, M_{2}, M_{3}, \ldots$ satisfying certain conditions and then show that 
$\left(b_{i}\right)=\left(f_{n_{i}}\right)$ satisfies the $\alpha+1$-step (for $\underline{\eta}$ ). We let $n_{i}=i$ for $i=1,2$ and set $M_{1}=N \sim\{1\}$. We now formulate the needed sub-lemma, analogous to Sub-Lemma 1.

Sub-Lemma 2. There exist positive integers $n_{1}, n_{2}, \ldots$ and infinite subsets of $N, M_{1}, M_{2}, \ldots$ with $n_{i}=i, i=1,2$, and $M_{1}=N \sim\{1\}$ so that, for all $s \geq 2$,

$$
\begin{aligned}
& n_{1}<\cdots<n_{s}<M_{s}, \\
& M_{s} \subset M_{s-1}, \\
& n_{s} \text { is the least element of } M_{s-1} .
\end{aligned}
$$

Given $1<r \leq s$, there is an open set $\mathscr{V} \subset \mathscr{U}$ and an $x_{2} \in \mathscr{V}$ so that

$$
\begin{aligned}
& \varphi\left(x_{2}\right)-\varphi\left(x_{1}\right)>(1-\eta) \delta, \\
& \sum_{1 \leq i<r}\left|f_{n_{i}}(t)-f\left(x_{1}\right)\right|<\eta \delta \text { for all } t \in \mathscr{V} \text {, } \\
& \sum_{r \leq i \leq s}\left|f_{n_{i}}(t)-f\left(x_{2}\right)\right|<\eta \delta \text { for all } t \in \mathscr{V}, \\
& \underline{\lambda} \leq \lambda<(1+\eta) \beta-\delta \text { where } \lambda=v_{\alpha}(\varphi)\left(x_{2}\right) \text {, } \\
& \left(f_{i}\right)_{i \in M_{s}} \text { satisfies the conclusion of Theorem } 4.2 \text { for the } \alpha \text {-case, } \\
& \text { with " } U \text { " }=\mathscr{V}, ~ " x "=x_{2} \text {. }
\end{aligned}
$$

That is, letting $n_{s+1}$ be the least element of $M_{s}$ and assuming $n_{s+1}=m_{1}<$ $m_{2}<\cdots$ is an infinite sequence in $M_{s}$, there exist $k, y_{1}, \ldots, y_{2 k} \stackrel{\mathrm{df}}{=} t$ in $\mathscr{V}$, and $\delta_{1}, \ldots, \delta_{k}>0$ so that

$$
\begin{array}{ll}
\text { (i) } & \varphi\left(y_{2 j}\right)-\varphi\left(y_{2 j-1}\right)>(1-\eta) \delta_{j} \text { for all } 1 \leq j \leq k, \\
\text { (ii) } & (1+\eta) \lambda>\sum_{j=1}^{k} \delta_{j}>(1-\eta) \lambda, \\
\text { (iii) } \sum_{\substack{i \in M_{s} \\
m_{j} \leq i<m_{j+1}}}\left|f_{i}(t)-f\left(y_{j}\right)\right|<\eta \delta_{\left[\frac{i+1}{2}\right]} \text { for all } j \leq 2 k-1, \\
\text { (iv) } \sum_{\substack{i \geq m_{2 k} \\
i \in M_{s}}}\left|f_{i}(t)-f(t)\right|<\eta \delta_{k} .
\end{array}
$$

Remark. Of course $x_{2}$ is given to us by Lemma 4.3 , i.e., by the statement containing (107)-(110). $\mathscr{V}$ is chosen after $x_{2}$ is picked.

Let us first show that Sub-Lemma 2 implies the main inductive step of Theorem 4.2; that is, $\left(b_{i}\right) \stackrel{\text { df }}{=}\left(f_{n_{i}}\right)$ works for $\alpha+1$. So, let $1<r<\ell_{1}<\ell_{2}<\cdots$ be an infinite sequence, and let $s=\ell_{1}-1$. So $s \geq 2$ and $n_{\ell_{1}}=n_{s+1}$. Now it follows from (112) and (113) that

$$
\left\{n_{s+1}, n_{s+2}, \ldots\right\} \subset M_{s} \text {. }
$$

Let $m_{j}=n_{\ell_{j}}$ for $j=1,2, \ldots$. Then by (119), $m_{1}, m_{2}, \ldots$ all belong to $M_{s}$. So we may choose $\mathscr{V} \subset \mathscr{U}$ and $x_{2} \in \mathscr{V}$ satisfying (114)-(117). Then 
there exist $k$ and $y_{1}, \ldots, y_{2 k} \stackrel{\mathrm{df}}{=} t$ in $\mathscr{V}$, and $\delta_{1}, \ldots, \delta_{k}>0$ satisfying (118). But then it follows that $\delta, \delta_{1}, \ldots, \delta_{k}$, and $x_{1}, x_{2}, y_{1}, \ldots, y_{2 k}$ satisfy the conclusion of 4.2 for $\alpha+1$. That is, if $k^{\prime}=k+1, \delta_{1}^{\prime}=\delta, \delta_{i}^{\prime}=\delta_{i-1}$, $2 \leq i \leq k^{\prime} ; x_{1}^{\prime}=x_{1}, x_{2}^{\prime}=x_{2}$, and $x_{j}^{\prime}=y_{j-2}$ for $3 \leq j \leq 2 k^{\prime}$, then $\delta_{1}^{\prime}, \ldots, \delta_{k^{\prime}}^{\prime}, x_{1}^{\prime}, \ldots, x_{2 k^{\prime}}^{\prime}$ satisfy (1)-(4) of Theorem 4.2. Indeed, (114) and (118i) yield (1).

As for (2)

$$
\begin{aligned}
\sum_{i=1}^{k^{\prime}} \delta_{i}^{\prime}= & \delta+\sum_{j=1}^{k} \delta_{j}>\delta+(1-\eta) \lambda \text { by }(118 \mathrm{ii}) \\
\geq & \delta+(1-\eta) \underline{\lambda} \text { by }(117) \\
> & (1-\eta)(\delta+\underline{\lambda})>(1-\eta)^{2} \beta \text { by }(1) \text { of } 3.9 \\
\delta+\sum_{j=1}^{k} \delta_{j} & <\delta+(1+\eta) \lambda \text { by }(118 \mathrm{ii}) \\
& <\delta+(1+\eta)((1+\eta) \beta-\delta) \text { by }(117) \\
& <(1+\eta)^{2} \beta
\end{aligned}
$$

Thus (2) of 4.2 holds, by (120) and (121), provided

$$
(1-\eta)^{2} \geq 1-\underline{\eta} \text { and }(1+\eta)^{2} \leq 1+\underline{\eta} \text {. }
$$

Finally, we verify (3) and (4). Let $m_{1}^{\prime}=1, m_{2}^{\prime}=r$, and $m_{j}^{\prime}=\ell_{j-2}$ for all $3 \leq j \leq k^{\prime}$. We have, since $t \in \mathscr{V}$, that

$$
\sum_{m_{j}^{\prime} \leq i<m_{j+1}^{\prime}}\left|f_{n_{i}}(t)-f\left(x_{j}^{\prime}\right)\right|<\eta \delta_{\left[\frac{i+1}{2}\right]}^{\prime}
$$

for all $1 \leq j \leq 2 k^{\prime}-1$. Indeed, (123) holds for $j=1,2$ by (115) and (116), and for $3 \leq j \leq 2 k+1$ by (118iii). Indeed, to see the latter, instead let $1 \leq j<2 k$; then

$$
\begin{aligned}
& \sum_{m_{j+2}^{\prime} \leq i<m_{j+3}^{\prime}}\left|f_{n_{i}}(t)-f\left(x_{j+2}^{\prime}\right)\right|=\sum_{\ell_{j} \leq i<\ell_{j+1}}\left|f_{n_{i}}(t)-f\left(y_{j}\right)\right| \\
& \quad=\sum_{m_{j} \leq n_{i}<m_{j+1}}\left|f_{n_{i}}(t)-f\left(y_{j}\right)\right| \text { since } m_{r}=n_{\ell_{r}} \text { for all } r \\
& \leq \sum_{m_{j} \leq i<m_{j+1}}\left|f_{i}(t)-f\left(y_{j}\right)\right|<\eta \delta_{\left[\frac{j+1}{2}\right]} \text { by }(118 \mathrm{iii}) \\
& =\eta \delta_{\left[\frac{i+3}{2}\right]}^{\prime} .
\end{aligned}
$$

Thus (3) holds (since $\eta<\underline{\eta}$ ). Finally, we have

$$
\sum_{i \geq m_{2 k^{\prime}}^{\prime}}\left|f_{n_{i}}(t)-f(t)\right|<\underline{\eta} \delta_{k+1}^{\prime}
$$

by (118iv) and (119). Hence (4) holds. 
We now give the proof of Sub-Lemma 2. We first complete the first step, i.e., the construction of $\mathscr{V}$ and $M_{2}$. (In this case, $s=2=r$.) First choose $x_{2} \in \mathscr{U}$ satisfying (107)-(110) for $m=2$. Then choose $\mathscr{V}$ an open neighborhood of $x_{2}$ with $\mathscr{V} \subset \mathscr{U}$, satisfying (115) and (116), using the continuity of $f_{1}$ and $f_{2}$. Finally by the induction hypothesis, choose $M_{2}$ infinite with $2<M_{2} \subset M_{1}$ so that (118) holds. We then have that (111), (112) hold, completing the proof for $s=2$.

Now suppose $s \geq 2$, and $n_{1}, \ldots, n_{s}$ and $M_{1}, \ldots, M_{s}$ have been constructed satisfying (111)-(118). It remains to carry out the construction for $s+1$. Let $n_{s+1}$ be the least element of $M_{s}$ and let $M^{1}=M_{s} \sim\left\{n_{s+1}\right\}$. We shall construct infinite sets $M^{1} \supset M^{2} \supset \cdots \supset M^{s+1}$ so that, for each $1<r \leq s+1$, there is an open set $\mathscr{V} \subset \mathscr{U}$ and an $x_{2} \in \mathscr{V}$ satisfying (114)-(117) (where we replace " $s$ " by " $s+1$ " in (116)) and (118) (where we replace " $M_{s}$ " by " $M^{r}$ " and " $n_{s+1}$ " by " $\ell_{r}$ " in (118)). Once this is done, the $s+1$-st step is complete, upon setting $M_{s+1}=M^{s+1}$, by virtue of Remark 1 after the statement of Theorem 4.2. So, let $1<r \leq s+1$ and suppose $M^{r-1}$ has been constructed. Now setting $m=n_{r}$, choose $x_{2} \in \mathscr{U}$ satisfying (107)-(110). Thus we have (114), (117), and thanks to (109) and (110)

$$
\sum_{1 \leq i<r}\left|f_{n_{i}}\left(x_{2}\right)-f\left(x_{1}\right)\right|<\eta \delta
$$

and

$$
\sum_{r \leq i \leq s+1}\left|f_{n_{i}}\left(x_{2}\right)-f\left(x_{2}\right)\right|<\eta \delta
$$

Thus we may choose an open $\mathscr{V} \subset \mathscr{U}$ with $x_{2} \in \mathscr{V}$ so that (115) and (116) hold for " $s$ " $=s+1$, by the continuity of $f_{n_{1}}, \ldots, f_{n_{s+1}}$. At last, by the induction hypothesis, choose $M^{r} \subset M^{r-1}$ so that (118) holds (replacing " $M_{s}$ " by " $M^{r}$ " in its statement).

This completes the inductive construction of the $M^{i}$ 's, hence the proof of Sub-Lemma 2, and thus the main inductive step of Theorem 4.2.

To finish the proof, let $1>\eta>0$ be given, $\beta>1$ a given countable ordinal, and suppose the theorem proved for all ordinals $\alpha<\beta$. If $\beta$ is a successor ordinal, we are done by the main inductive step. Otherwise, let $\mathscr{U}$ be an open neighborhood of $x$, choose $1>\eta>0$ with

$$
(1-\eta)^{2} \geq 1-\underline{\eta}, \quad(1+\eta)^{2} \leq 1+\underline{\eta},
$$

and now, by the definition of $v_{\beta}(\varphi)(x)$, choose $x^{\prime}$ in $\mathscr{U}$ and $\alpha<\beta$ with

$$
\left\{\begin{array}{l}
(1-\eta) v_{\beta}(\varphi)(x)<\lambda<(1+\eta) v_{\beta}(\varphi)(x) \\
\text { where } \lambda=v_{\alpha}(\varphi)\left(x^{\prime}\right) .
\end{array}\right.
$$

Now choose $\left(b_{j}\right)$ a subsequence of $\left(f_{j}\right)$ satisfying the conclusion of Theorem 4.2 (for $\eta$ and $\alpha$ ). Finally, given $1=m_{1}<m_{2}<\cdots$ and choosing $k$, the 
$x_{i}$ 's and $\delta_{i}$ 's as in the statement of 4.2 , we have

$$
\begin{aligned}
(1-\underline{\eta}) v_{\beta}(\varphi)(x) & \leq(1-\eta)^{2} v_{\beta}(\varphi)(x) \text { by }(126) \\
& <(1-\eta) \lambda \text { by }(127) \\
& <\sum_{i=1}^{k} \delta_{i}<(1+\eta) \lambda \text { by }(2) \text { of Theorem } 4.2 \\
& <(1+\eta)^{2} v_{\beta}(\varphi)(x) \text { by }(127) \\
& \leq(1+\underline{\eta}) v_{\beta}(\varphi)(x) \text { by }(126) .
\end{aligned}
$$

Thus (2) of 4.2 holds (for " $n=\underline{\eta}$ and " $\lambda$ " $=v_{\beta}(\varphi)(x)$ ), and (1), (3), and (4) hold since $\eta<\underline{\eta}$. This completes the entire proof.

\section{REFERENCES}

[Be] S.F. Bellenot, More quasi-reflexive subspaces, Proc. Amer. Math. Soc. 101 (1987), 693-696.

[Bes-P] C. Bessaga and A. Pelczyński, On bases and unconditional convergence of series in Banach spaces, Studia Math. 17 (1958), 151-164.

[Bo-De] J. Bourgain and F. Delbaen, $A$ class of special $\mathscr{L}^{\infty}$-spaces, Asta Math. 145 (1980), 155-1 76.

[Bo-R] J. Bourgain and H.P. Rosenthal, Geometrical implications of certain finite dimensional decompositions, Bull. Soc. Math. Belg. 32 (1980), 57-82.

[Do] L.E. Dor, On sequences spanning a complex $\ell^{1}$ space, Proc. Amer. Math. Soc. 47 (1975), 515-516.

[E] J. Elton, Weakly null normalized sequences in Banach spaces, Doctoral Thesis, Yale University, 1978.

[F] C. Finet, Subspaces of Asplund Banach spaces with the point continuity property, Israel J. Math. 60 (1987), 191-198.

[Go] W.T. Gowers, $A$ space not containing $c_{0}, \ell_{1}$ or a reflexive subspace, preprint.

[HOR] R. Haydon, E. Odell, and H. Rosenthal, On certain classes of Baire-1 functions with applications to Banach space theory, Functional Analysis Proceedings (The University of Texas at Austin 1987-89), Lecture Notes in Math., vol. 1470, Springer-Verlag, Berlin and New York, 1991, pp. 1-35.

[JR] W.B. Johnson and H. Rosenthal, On $\omega^{*}$-basic sequences and their applications to the study of Banach spaces, Studia Math. 43 (1972), 77-92.

[KL] A.S. Kechris and A. Louveau, A classification of Baire class 1 functions, Trans. Amer. Math. Soc. 318 (1990), 209-236.

[OR] E. Odell and H. Rosenthal, $A$ double-dual characterization of separable Banach spaces containing $\ell^{1}$, Israel J. Math. 20 (1975), 375-384.

[P1] A. Pelczyński, A connection between weakly unconditional convergence and weak completeness of Banach spaces, Bull. Acad. Polon. Sci. 6 (1958), 251-253.

[P2] Banach spaces on which every unconditionally converging operator is weakly compact, Bull. Acad. Polon. Sci. 10 (1962), 641-648.

[R1] H. Rosenthal, A characterization of Banach spaces containing $\ell^{1}$, Proc. Nat. Acad. Sci. U.S.A. 71 (1974), 2411-2413.

[R2] - Some recent discoveries in the isomorphic theory of Banach spaces, Bull. Amer. Math. Soc. 84 (1978), 803-831.

[R3] —, Weak*-Polish Banach spaces, J. Funct. Anal. 76 (1988), 267-316. 
[R4] - Some aspects of the subspace structure of infinite dimensional Banach spaces, Approximation Theory and Functional Analysis (C. Chui, ed.), Academic Press, New York, 1991, pp. 151-176.

[R5] - Differences of bounded semi-continuous functions (in preparation).

[R6] - Boundedly complete weak-Cauchy basic sequences in Banach spaces with the PCP (to appear).

ABSTRACT. A subsequence principle is obtained, characterizing Banach spaces containing $c_{0}$, in the spirit of the author's 1974 characterization of Banach spaces containing $\ell^{1}$.

Definition. A sequence $\left(b_{j}\right)$ in a Banach space is called strongly summing (s.s.) if $\left(b_{j}\right)$ is a weak-Cauchy basic sequence so that whenever scalars $\left(c_{j}\right)$ satisfy $\sup _{n}\left\|\sum_{j=1}^{n} c_{j} b_{j}\right\|<\infty$, then $\sum c_{j}$ converges.

A simple permanence property: if $\left(b_{j}\right)$ is an (s.s.) basis for a Banach space $B$ and $\left(b_{j}^{*}\right)$ are its biorthogonal functionals in $B^{*}$, then $\left(\sum_{j=1}^{n} b_{j}^{*}\right)_{n=1}^{\infty}$ is a non-trivial weak-Cauchy sequence in $B^{*}$; hence $B^{*}$ fails to be weakly sequentially complete. (A weak-Cauchy sequence is called non-trivial if it is non-weakly convergent.)

Theorem. Every non-trivial weak-Cauchy sequence in a (real or complex) Banach space has either an (s.s.) subsequence or a convex block basis equivalent to the summing basis.

Remark. The two alternatives of the theorem are easily seen to be mutually exclusive.

Corollary 1. A Banach space $B$ contains no isomorph of $c_{0}$ if and only if every non-trivial weak-Cauchy sequence in $B$ has an (s.s.) subsequence.

Combining the $c_{0}$ - and $\ell^{1}$-Theorems, we obtain

Corollary 2. If $B$ is a non-reflexive Banach space such that $X^{*}$ is weakly sequentially complete for all linear subspaces $X$ of $B$, then $c_{0}$ embeds in $B$; in fact, $B$ has property $(u)$.

The proof of the theorem involves a careful study of differences of bounded semi-continuous functions. The results of this study may be of independent interest.

Department of Mathematics, The University of Texas at Austin, Austin, TeXas 78712-

E-mail address: rosenthlemath.utexas.edu 